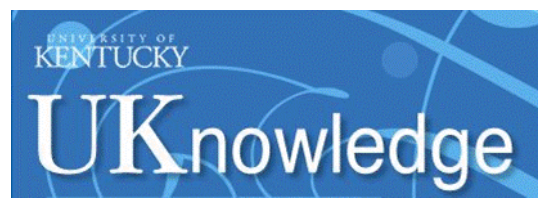

University of Kentucky

UKnowledge

Chemical and Materials Engineering Faculty

Publications

Chemical and Materials Engineering

7-2017

\title{
Size and Shape Distributions of Primary Crystallites in Titania Aggregates
}

\author{
Eric A. Grulke \\ University of Kentucky, eric.grulke@uky.edu \\ Kazuhiro Yamamoto \\ National Institute of Advanced Industrial Science and Technology, Japan \\ Kazuhiro Kumagai \\ National Institute of Advanced Industrial Science and Technology, Japan \\ Ines Häusler \\ Federal Institute for Materials Research and Testing, Germany \\ Werner Österle \\ Federal Institute for Materials Research and Testing, Germany \\ Follow this and additional works at: https://uknowledge.uky.edu/cme_facpub
}

Part of the Chemical Engineering Commons, Materials Science and Engineering Commons, See nextpage for additional authors

Right click to open a feedback form in a new tab to let us know how this document benefits you.

\section{Repository Citation}

Grulke, Eric A.; Yamamoto, Kazuhiro; Kumagai, Kazuhiro; Häusler, Ines; Österle, Werner; Ortel, Erik; Hodoroaba, Vasile-Dan; Brown, Scott C.; Chan, Christopher; Zheng, Jiwen; Yamamoto, Kenji; Yashiki, Kouji; Song, Nam Woong; Kim, Young Heon; Stefaniak, Aleksandr B; Schwegler-Berry, D.; Coleman, Victoria A.; Jämting, Åsa K.; Herrmann, Jan; Arakawa, Toru; Burchett, Woodrow W.; Lambert, Joshua W.; and Stromberg, Arnold J., "Size and Shape Distributions of Primary Crystallites in Titania Aggregates" (2017). Chemical and Materials Engineering Faculty Publications. 58.

https://uknowledge.uky.edu/cme_facpub/58

This Article is brought to you for free and open access by the Chemical and Materials Engineering at UKnowledge. It has been accepted for inclusion in Chemical and Materials Engineering Faculty Publications by an authorized administrator of UKnowledge. For more information, please contact UKnowledge@lsv.uky.edu. 


\section{Size and Shape Distributions of Primary Crystallites in Titania Aggregates}

\section{Digital Object Identifier (DOI)}

https://doi.org/10.1016/j.apt.2017.03.027

\section{Notes/Citation Information}

Published in Advanced Powder Technology, v. 28, issue 7, p. 1647-1659.

(C) 2017 Published by Elsevier B.V. on behalf of The Society of Powder Technology Japan. All rights reserved.

This manuscript version is made available under the CC-BY-NC-ND 4.0 license

http://creativecommons.org/licenses/by-nc-nd/4.0/.

The document available for download is the author's post-peer-review final draft of the article.

\section{Authors}

Eric A. Grulke, Kazuhiro Yamamoto, Kazuhiro Kumagai, Ines Häusler, Werner Österle, Erik Ortel, Vasile-Dan Hodoroaba, Scott C. Brown, Christopher Chan, Jiwen Zheng, Kenji Yamamoto, Kouji Yashiki, Nam Woong Song, Young Heon Kim, Aleksandr B Stefaniak, D. Schwegler-Berry, Victoria A. Coleman, Åsa K. Jämting, Jan Herrmann, Toru Arakawa, Woodrow W. Burchett, Joshua W. Lambert, and Arnold J. Stromberg 


\section{Size and shape distributions of primary crystallites in titania aggregates}

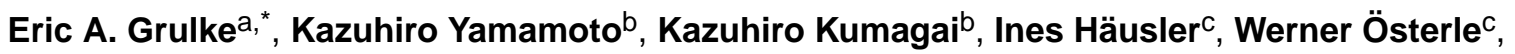
Erik Ortel ${ }^{\mathrm{c}}$, Vasile-Dan Hodoroaba ${ }^{\mathrm{c}}$, Scott C. Brown ${ }^{\mathrm{d}}$, Christopher Chan ${ }^{\mathrm{e}}$, Jiwen Zheng ${ }^{\dagger}$, Kenji Yamamoto ${ }^{g}$, Kouji Yashiki ${ }^{g}$, Nam Woong Songh ${ }^{\mathrm{h}}$, Young Heon Kim ${ }^{\mathrm{h}}$, Aleksandr B. Stefaniak', D. Schwegler-Berry', Victoria A. Coleman', Åsa K. Jämting', Jan Herrmann', Toru

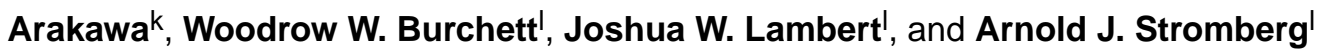

${ }^{a}$ Chemical \& Materials Engineering, University of Kentucky, Lexington, KY, USA

bNational Institute of Advanced Industrial Science and Technology (AIST), Tsukuba, Japan

'Federal Institute for Materials Research and Testing (BAM), Berlin, Germany

dThe Chemours Company, Wilmington, DE, USA

eDuPont Central Research and Development, Wilmington, DE, USA

fU.S. Food and Drug Administration, Silver Springs, MD, United States

glshihara Sangyo Kaisha, Ltd., Osaka, Japan

hKorea Research Institute of Standards and Science (KRISS), Daejeon, Republic of Korea

iU.S. National Institute for Occupational Safety and Health, Morgantown, WV, United States

jNational Measurement Institute, Lindfield, NSW, Australia

kTayca Corporation, Osaka, Japan

'Applied Statistics Laboratory, University of Kentucky, Lexington, KY, USA

\section{Abstract}

The primary crystallite size of titania powder relates to its properties in a number of applications. Transmission electron microscopy was used in this interlaboratory comparison (ILC) to measure primary crystallite size and shape distributions for a commercial aggregated titania powder. Data of four size descriptors and two shape descriptors were evaluated across nine laboratories. Data repeatability and reproducibility was evaluated by analysis of variance. One-third of the laboratory pairs had similar size descriptor data, but $83 \%$ of the pairs had similar aspect ratio data. Scale descriptor distributions were generally unimodal and were well-described by lognormal reference models. Shape descriptor distributions were multi-modal but data visualization plots demonstrated that the Weibull distribution was preferred to the normal distribution. For the equivalent circular diameter size descriptor, measurement uncertainties of the lognormal distribution scale and width

"Corresponding author. eric.grulke@uky.edu (E.A. Grulke).

Appendix A. Supplementary material: Supplementary data associated with this article can be found, in the online version, at http:// dx.doi.org/10.1016/j.apt.2017.03.027. 
parameters were $9.5 \%$ and $22 \%$, respectively. For the aspect ratio shape descriptor, the measurement uncertainties of the Weibull distribution scale and width parameters were $7.0 \%$ and $26 \%$, respectively. Both measurement uncertainty estimates and data visualizations should be used to analyze size and shape distributions of particles on the nanoscale.

\section{Keywords}

Measurement uncertainty; Size distribution; Shape distribution; TEM; Titania

\section{Introduction}

This section reviews particle size and shape distributions by transmission electron microscopy (TEM), stakeholder needs for this information, morphology descriptions of powder aggregates, the relevance of primary crystallite size and shape distributions for titania applications, and the project objectives.

\subsection{Size and shape distributions by transmission electron microscopy}

While many of the measurements methods for particle sizes in the nanoscale have focused on assessing an average particle size, the performance properties of nanoparticles often depend on size and shape distributions. Indeed, the nanoparticle size distribution is important to product performance in applications, in the environment, and for health, safety, and regulatory issues. Transmission electron microscopy (TEM) is a standard method for determining nanoparticle sizes.

This case study provides a scientific foundation for an International Organization for Standardization (ISO; www.iso.org) standard for the measurement of particle size distributions on the nanoscale by TEM. The specific ISO committee is ISO/TC229 Nanotechnologies, which was formed in 2005 and has 34 national member bodies, 40 liaison members (other ISO technical committees or international organizations) and 11 observers. The authors of this study include members of Joint Working Group 2 (JWG2), Measurements and Methods. This particular project is a consensus choice of JWG2 as an example of aggregated particle size and shape distributions. ISO standards exist for the graphical representation of particle size distributions [1], calculation of average size and moments [2], fitting reference models to distribution data [3], logarithmic normal probability distributions [4], descriptors for particle size and shape [5], accuracy of measurement methods [6,7], and static image analysis methods [8]. These methods have been applied to measurements made for this project. The interlaboratory comparison team includes four national metrology institutes, three titania manufacturing companies, two regulatory agencies, and a university.

Although transmission electron microscopy (TEM) has been extensively applied to characterize nanomaterials, standard methods for imaging, analyzing and reporting size distributions are lacking. Exceptions to this circumstance are the average particle sizes and the associated measurement uncertainties for TEM analyses of reference materials [9] and certificated reference materials [10]. Nanomaterial and nanoparticle products are moving 
toward, or are in, the marketplace. Commercial and regulatory stakeholders will need guidance on measurement methods and their measurement uncertainties when evaluated by multiple laboratories. Classical analysis methods are available for particle size and particle size uncertainties [11-14]. A semi-automated image analysis method has reported size distribution statistics from an interlaboratory comparison (ILC) [15] of gold reference material samples [9]. Here, a more realistic, commercial sample of nanoscale titanium dioxide in an aggregated/agglomerated state is analyzed using manual image analysis methods.

\subsection{Stakeholder needs for size and shape distribution data}

Size and shape distribution measurements and analyses of titania powders are needed by multiple stakeholders, e.g., academia, industry, government and the public at large. Titania powder performance properties have been related to their physico-chemical characteristics, including size, shape, surface structure and surface texture. In this work, the TEM measurements are not compared to traditional, one-point estimates for particle size, such as $\mathrm{x}$-ray diffraction (XRD) or specific surface area (BET) analysis. Neither method, XRD or BET, can provide information about particle shape. Our methods report the primary crystallite size and shape distribution, estimate parameters of references distributions fitted to the data, compute measurement uncertainties of these parameters, and visualize the correspondence between the data and the fitted reference distributions.

This protocol was developed based on an interlaboratory comparison (ILC) study that conformed to guidelines established by the Versailles Project on Advanced Materials and Standards (VAMAS) [16] and ISO 5725 [17]. Key needs of the International Standards stakeholder and user community include: (1) measurement of 'real life' materials, (2) highly automated protocol steps, including image acquisition, particle capture, data quality assessments, (3) comparison of data to reference distribution models, (4) measurement uncertainty assessments for evaluations by different laboratories, and (5) data visualization tools to compare methods, procedures, and descriptors. JWG2 of ISO/TC229 has established five ILC case studies for a broad spectrum of particle types. These include: unimodal, discrete spheroidal nanoparticles (gold), a bimodal mixture of discrete nanoparticles (colloidal silicas), a discrete nanoparticle mixture with different shapes (gold nanorods), amorphous aciniform aggregates (carbon black), and aggregates of primary crystallites (titania). The protocol provides an example of determining size and shape descriptors by manually outlining aggregated primary crystallites with clearly defined edges [18]. The approach is based on methods reported for titania powder synthesis research plus methods in use by titanium dioxide manufacturers.

\subsection{Morphologies of powder aggregates}

A recent study [19] has helped identify differences of the internal morphologies of powder aggregates in the categories, amorphous (silica gel), paracrystalline (carbon black), crystalline and amorphous (siliceous earth and organic clay), amorphous shell over crystalline core (silica-coated titania), and crystalline aggregates (iron oxide, fumed alumina, calcium carbonate and titania). While the term, primary particle, has been used to describe 
the individual elements fused together in titania aggregates [20], 'primary crystallite' is a more precise term as there are grain boundaries between these elements [19].

\subsection{Relevance of size and shape distributions for titania applications}

Aggregate particle size distributions are frequently measured via non-microscopy methods, such as those that measure hydrodynamic particle size (e.g., centrifugal liquid sedimentation); these have been the subject of multiple interlaboratory comparisons in the past. Here, the focus is on the measurement of size and shape distributions of primary crystallites in a titanium dioxide sample. This titania was a commercial powder sample consisting of primary crystallites aggregated to micron-scale particles. The sizes and shapes of the primary crystallites are known to link with the performance of titania, as shown in Table 1. In many of these applications, the size and shape of the primary crystallites of the titania aggregate are essential to product performance rather than the size and shape of the aggregate. Titania's primary crystallite size has been linked to its performance as a catalyst [21-25], as a photocatalyst [26,27], in photooxidation [28,29], and in cytotoxicity tests [3032] [33]. Particle shape has also been linked to the performance of titania in optical applications [34-36]. Primary crystallite grain shapes vary and there have been recent reports of specific shapes affecting titania performance in new applications [37-39].

The primary crystallites of titania aggregates are tightly fused and it is not reasonable to use mechanical action to release primary crystallites for direct measurement [40]. In addition, as-manufactured titania products can have residual acidic or basic impurities on their surfaces [41] or surface coatings of some type. For use in consumer or commercial products, metal oxides are often stabilized with inorganic salts [42] and polymers [43], or functionalized with other ligands such as alkoxysilanes [44]. In this study, an inorganic salt, sodium hexametaphosphate was used as a dispersant so that TEM grids could be prepared using dilute aqueous dispersions of stabilized titania powders.

\subsection{Project objectives}

Aggregated/agglomerated powder samples are representative of many commercial materials and mirror the majority of TEM sample preparation protocols. The measurement uncertainty of titania primary crystallite size and shape parameters via an interlaboratory comparison has not been reported to our knowledge. Current size measurement for similar powders may not be based on well-established protocols and the uncertainty in measurement is often not conveyed. The lack of confidence in reported values makes it difficult to draw reliable correlations between primary crystallite size and the behavior of various titania in their applications.

The protocol for this sample includes sample preparation, instrument factors, image capture, particle analysis, and data analysis, which has been subdivided into raw data triage, repeatability/reproducibility assessment, fitting distributions to data, estimating measurement uncertainty of distribution parameters, and visualization of results. This protocol is generally applicable to measuring primary crystallites of aggregated titania, but is not necessarily optimized for specific titania powder samples or specific titania application needs. The data analysis elements and statistics tools reported here can be used to improve 
elements of the protocol, leading to lower measurement uncertainty values, for example.

Statistical protocols are used to distinguish between datasets, to select descriptors with similar means, to fit reference models to distribution data, and to compare two-dimensional distributions. While measurement uncertainty is an important metric by which to judge data quality, visualization tools can provide additional information. Rather, measurement uncertainty estimates should be used along with visualization tools that can compare model predictions to the data. Our general approach can be used by stakeholders for evaluating descriptors of particle size and shape distributions of commercial materials.

\section{Materials and methods}

This section provides an overview of the protocol's sample preparation, instrument factors and image acquisition, particle analysis, and data analysis methods. Additional details on the protocol steps, background data, and definitions are provided in the Supplemental Material associated with this article.

\subsection{Sample preparation}

The sample is a commercial titanium dioxide material (MT-500BW, rutile, supplied by Tayca), which could be used for the applications cited in Table 1. This powder sample has aggregates with diameters greater than one micron and composed of fused primary crystallites with length scales in the range of 30-50 nm. One laboratory prepared and distributed all the TEM sample grids for this study. The material was dispersed by ultrasonication in an aqueous solution of sodium hexametaphosphate [CAS Reg. number 10124-56-8, (NaPO3)6], a common approach for dispersing titanium dioxide particles in aqueous media. Ultrasonication generates smaller aggregates (shown in the upper frame of Fig. 1), which have smaller depths of field when deposited on the TEM support. Smaller aggregates make it easier to focus on and image the primary crystallites (shown in the lower frame of Fig. 1). The dispersion process was deemed adequate to disperse aggregates on TEM grids for image analysis and was not optimized further. More details on the dispersion process are provided in Supplemental Material.

\subsection{Instrument factors, image acquisition}

Each collaborating laboratory used different TEM instruments. TEM operating conditions are shown in the Supplementary Material, Section S3, along with calibration protocols. For a nominal crystallite diameter of $20 \mathrm{~nm}$, a resolution of $0.5 \mathrm{~nm} /$ pixel gives a length uncertainty of $1.6 \%$ (the error introduced by miscounting one pixel). Smaller crystallites would have higher length uncertainties. Primary crystallites were trace manually and saved. Only particles with clear and distinguishable edges were traced. Operators selected images with appropriate contrast between particle and background and there were no automated thresholding issues for these samples. No issues were reported with respect to brightness/ contrast. Additional details on image capture and particle analysis are shown in Supplementary Material, Section 4.

The primary crystallites of this commercial titania sample cannot be separated physically into individual nanoparticles. Manual particle tracing was used with the traced images being 
saved for each frame. Only those particles that have clear and distinguishable edges or boundaries were reported. This protocol assumes that all images were taken in digital format. Procedure steps were provided for the open source software, ImageJ (http:// rsbweb.nih.-gov/ij/download.html). Three sets of panels are shown in Fig. 2; the yellow rectangles in the right hand frame of the panels in the top row shows some possible selections of particles for capture. The right hand panel in the middle row shows a primary crystallite with easily distinguishable edges that has been manually traced (yellow boundary). The right hand panel in the lowest row shows additional examples of particles that should be captured (yellow boundaries). Fig. 3 shows an example of an object that does not have clearly distinguishable edges and should not be captured. There is likely to be a line of fusion between the two segments of the object, but the line of demarcation is not obvious.

\subsection{Size and shape descriptors}

There are a number of size and shape descriptors that could have been used for this study (see [5] for a complete list). The size descriptors can be used to compute shape descriptors. Size descriptors reported by all laboratories were area $\left(\mathrm{nm}^{2}\right)$, maximum Feret diameter $(\mathrm{nm})$, minimum Feret diameter (nm), and equivalent circular diameter (ECD, nm; Eq. (1)). Two shape descriptors, aspect ratio (minFeret/Feret) and compactness (ECD/Feret), were computed from these size descriptors (Eqs. (2) and (3), respectively). Equations for ECD, aspect ratio, and compactness are:

$$
\begin{aligned}
\mathrm{ECD} & =\sqrt{\frac{4 \cdot A}{\pi}} \\
\text { aspect ratio } & =\frac{\operatorname{minFeret}}{\operatorname{maxFeret}} \\
\text { compactness } & =\frac{\mathrm{ECD}}{\text { maxFeret }}
\end{aligned}
$$

The descriptor definitions follow ISO 9276-6 [5] while the statistical definitions follow ISO 5725 [17]. Generally, ISO prefers shape descriptors with values between zero to one. Other descriptor and statistical definitions are provided in the Supplementary Material, Section S6. The use of manual particle outlining eliminated touching particle issues, which often occur for discrete particles dispersed on TEM supports.

Measured areas are based on the number of pixels associated with the primary crystallites. To reduce area measurement errors for a circle to less than 5\%, the recommended pixel numbers per particle range from 100 to 200 (ISO 9276-6). At a specific magnification level, the number of pixels per nanometer can be estimated, which allows estimates of the number 
of pixels per particle. Thus, it is often possible to determine, during the image capture step, whether the error on smaller particles is sufficient, and adjust the magnification if needed.

\subsection{Data analysis}

Descriptor data can be analyzed directly, either by comparing grand mean values and standard deviations to individual datasets using one-way ANOVA or by comparing datasets pair-wise to determine whether the sample population means were statistically similar. The conventional statistic is the $\mathrm{p}$-value. These methods provide qualitative dataset comparisons, addressing the repeatability (intralaboratory) or reproducibility (interlaboratory) of the data. Bivariate analysis can be used to compare descriptor pairs between two different datasets; the analysis returns a p-value and an energy measure [46,47]. Both are qualitative measures of statistical similarity.

Cumulative distributions were constructed for each descriptor in a dataset by sorting them in numerical order and assigning a cumulative frequency fraction value to each point. Reference models (normal, lognormal, or Weibull) can be fitted to the cumulative distribution data; the resulting scale and breadth parameter with their standard errors can then be compared either across the grand dataset (ANOVA) or pair-wise (pair-wise ANOVA). Three copyrighted programs have been developed, programmed in $\mathrm{R}$ and implemented as Shiny applications, to provide consistent statistical analyses across the ILC. They are: ANOVA, https://shiny.as.uky.edu/anova-app/; curve fitting, https:// shiny.as.uky.edu/curve-fitting-app/; and bivariate analysis, https://shiny.as.uky.edu/bivariate-fitting-app/.

Table 2 summarizes the data elements analyzed, the measurement objectives, the specific metrics, and the statistical methods used in this study.

2.4.1. Comparison of descriptor datasets-The industrial sample used in this ILC is not a certified reference material, so it is not possible to compute the bias or the relative bias of the datasets. We can determine the grand mean, $\bar{\chi}$, and standard deviation, s, of descriptors across all datasets, and then find a relative coefficient of variation (reported as a percentage).

$$
C_{v}=\frac{S}{\bar{\chi}}
$$

The relative coefficient of variation is defined as the ratio of the standard deviation, s, of the descriptor means for all datasets divided by the grand mean, $\bar{\chi}$, of the descriptor and is reported as a percentage (Eq. (4)).

2.4.2. Comparison of descriptor distributions-Single descriptor distributions were compared with two methods. Pair-wise comparison of the experimental cumulative distributions was done using bivariate analysis [46,47]. This method does not rely on fitting reference models to data and has value when the descriptor distribution does not conform to 
a selected model distribution, making it applicable to multimodal data. The second method fitted statistical reference models to distribution data, reporting estimated scale and width parameters obtained by minimizing the error between model and data. The parameter estimates and their standard errors, from the second technique, can be converted directly to coefficients of variation for scale and width parameters (Eq. (4)). These are then converted to expanded measurement uncertainty values for the fitted parameters (Eq. (5)):

$$
U_{\mathrm{ILC}}=k \cdot C_{v} \cdot \sqrt{1+1 / n}
$$

where $U_{I L C}$ is the relative expanded measurement uncertainty, the coverage factor $\mathrm{k}$ is taken as 2, and $\mathrm{n}$ is the number of observations [48]. A preferred model should be selected on both the quality of the parameter estimates (low coefficients of variation are preferred) and the visualizations of the fit, including a histogram, the cumulative distribution, and relative residual differences between the model and the data.

2.4.3. Comparison of descriptor-descriptor distributions-The comparison of single descriptor distributions does not reveal potential variations in morphology, which might better be addressed by comparing size-size or size-shape distributions. These distributions can be compared using the bivariate analysis method. This has value as it reflects potential differences in morphology without relying on adherence to specific distribution models.

\section{Results and discussion}

The major objective of this ILC is to report data quality effects for a standard protocol for measuring particle size and shape distributions of primary crystallites in a commercial titania powder. There are four major elements of the study: evaluating the quality of the raw data using ANOVA and/or bivariate analysis, generating reference model parameter values by fitting size and shape descriptor distribution data to reference models, using these values to estimate measurement uncertainties of these fitted parameters, and visualizing differences between data and model. Consumer, industrial, and regulatory stakeholders can follow this method to produce, report, and evaluate particle size and shape distributions by TEM.

\subsection{Data quality: effects of protocol factors}

Several factors might be responsible for inter-laboratory variations. These include: the instrument used (TEM or STEM), the number of particles analyzed, the number of frames analyzed, the calibration method (gold nanoparticles, gratings, MAG*I*CAL ${ }^{\circledR}$ [49], or other), the calibration scale (nm per pixel), and the software used for analysis (ImageJ or other). Table 3 lists experimental factors that were varied in this study for the eleven different data-sets. Laboratories were to report results for a minimum of 500 particles and there was no requirement set on the number of frames to be used. Multiple calibration methods were reported and three different image capturing software programs were used. Image resolution varied by an order of magnitude, from 0.15 to $1.4 \mathrm{~nm} /$ pixel.

Adv Powder Technol. Author manuscript; available in PMC 2017 December 01. 
3.1.1. Effects of factors on reproducibility of descriptors-This section addresses the effects of non-instrument factors on the reproducibility of descriptors. The one-way ANOVA compares the descriptor means of individual datasets (the datasets from each lab) to the grand mean of all the data for that descriptor. When the value of the statistic, $p$, is greater than 0.05 , the null hypothesis cannot be rejected, i.e., the assumption that the mean of one data-set is the same as that of the grand mean cannot be rejected.

Seven factors, the laboratory doing the analysis, the number of particles reported, the instrument manufacturer (shown in the Supplementary Material, Table S2), the number of frames used, the calibration method, the resolution value ( $\mathrm{nm} / \mathrm{pixel})$, and the imaging software, were compared to the scale and shape descriptor datasets using ANOVA. For five of the six descriptors, $p<0.05$ for the full ILC data: more than one laboratory had means different from the grand mean. An example of the ANOVA boxplot for the Feret diameter descriptor is shown in the Supplementary Material (Fig. S9).

Two factors, the calibration method and the software used, had some descriptors that conformed to the null hypothesis as shown in Table 4. For example, the equivalent circular diameter and the minimum Feret diameter had $p$-values greater than 0.05 when the calibration method was used the group variable. This is interpreted to mean that the calibration method was not a factor in the grand means of ECD and minFeret. When software was the group variable, Feret, minFeret, ECD, and aspect ratio had p-values greater than 0.05; this is interpreted to mean that the imaging software is not a factor in the grand means of these descriptors. Since the factors, instrument manufacturer, numbers of particles analyzed, number of frames analyzed, and scale (nm per pixel) values, were dependent on the reporting laboratories, ranges of these factors that would result in $\mathrm{p}$ values greater than 0.05 could not be determined from the data.

The number of particles reported varied by a factor of 2 while the number of frames used varied by an order of magnitude. Each factor impacted reproducibility, but it was not possible to interpret in what ways this might be occurring using ANOVA. However, an individual lab could use one-way or pair-wise ANOVA to assess this for their samples and procedures; this constitutes an intralaboratory repeatability evaluation. In general, when there are only a few frames, each frame may more closely resemble the total sample population. Conversely, when there are many frames, there can be more frame-to-frame variation in the frame means.

3.1.2. Minimum area of reported particles-In some of the particle size and shape distribution case studies organized by JWG2, there were significant numbers of undersized particles, i.e., particles which had a pixel count less than that recommended for precision[3]. The resolution, nm per pixel, is used to compute the area for each pixel. This value times 200 gives the suggested minimum particle area (the error in the area measurement will be $\sim 5 \%$ at this number; [3]). Across the ILC, 5809 particles were reported with areas ranging from 20 to $15,460 \mathrm{~nm}^{2}$. Table 5 shows the area at $5 \%$ measurement error for each laboratory and that laboratory's minimum reported area. None of the laboratories reported particles that had areas lower than the recommended number of pixels at their calibration scales. 
The grand mean average scale and width for the area distribution can be used to estimate what percentage of particles might be 'missed' for each laboratory at the 'edges' of the distribution. Given that the resolution between laboratories varies by an order of magnitude, it was possible that labs with high resolution values might miss significant cumulative fractions of small particles. Table 5 shows, for all laboratories, the primary crystallite area at the 200 pixel cutoff, the minimum area reported by the lab, and an estimate of the cumulative percent of primary crystallites that would not have been reported by the specific lab. This analysis assumes that the grand scale and width values for the ILC represent the true population. As shown in Table 5, the highest estimate for missed particles was $0.3 \%$ for L8. Therefore, the magnifications used by all labs in this ILC appear to have included appropriate particles. Should this not have been the case, images at higher magnifications would be needed to properly count small particles. With this method, the number of 'nonparticles' would be very low, if not zero.

3.1.3. Effect of instrument type-Two laboratories performed tests with both TEM and STEM (SEM in transmission mode; [50-52]) instruments. While lateral resolution is better for TEMs than for SEMs and the voltage used in SEMs is lower than in TEMs, the technique is quite similar to STEM. Laboratories L1 and L2 reported data (four size descriptors: area, Feret, minFeret, and equivalent circular diameter; two shape descriptors: aspect ratio and compactness) for the prepared samples using both TEM and STEM instruments. These data were labeled: L1 (TEM) and L1a (STEM), and L2 (TEM) and L2a (STEM), respectively. The TEM data were pooled (coded, L1 and L2) and the STEM data were pooled (coded L1a and L2a). The pooled sets were compared using one-way ANOVA Only the Feret diameter was found to meet the null hypothesis by this analysis, giving a mean Feret diameter of (50.0 $\pm 19.1) \mathrm{nm}$. The other five descriptors had different means for the two instrument methods. For all descriptors, the TEM value was larger than the STEM value. The ratios of STEM to TEM values are in the range of $0.93-0.96$, representing a 4-7\% difference. Slightly different results were obtained when TEM and STEM data from the same sample were compared, specifically, L1 with L1a and L2 with L2a. In the first instance, the Feret diameters were statistically similar but the minFeret diameters were not. The opposite was the case for lab L2; the Feret diameters were dissimilar and the minFeret diameters were similar. Therefore, the aspect ratios within these pairs were dissimilar. Better understanding of these differences may be pursued in future work.

\subsection{Pair-wise analysis of descriptors}

Industrial users may wish to compare distributions of unknown samples to a standard sample directly, without referring to reference distributions. Pair-wise ANOVA, done using a commercial statistical package (Systat ${ }^{\circledR}$ v13.1), can be used to identify pairs of datasets that are dissimilar. If the statistic, $p$, has a value less than 0.05 , the null hypothesis is rejected. Bivariate analysis also compares descriptors of two datasets. The bivariate analysis tool generates a pseudo population by developing a 'joint' population for the two datasets being compared, and then determines whether the two initial datasets are dissimilar from the generated population. The bivariate method is independent of reference models and can be applied directly to single descriptor cumulative distributions (the aspect ratio cumulative 
distribution for example), or size-shape distributions, as visualized by a two-dimensional plot of a size descriptor against a shape descriptor.

When a number of laboratories are generating particle size distributions that are different from the grand mean as assessed by ANOVA, it can useful to find pairs of laboratories that have similar means. Pair-wise ANOVA analysis can be used to determine which descriptor datasets have similar means. For size descriptors, one-third of the lab pairs with similar means (Table 6). The aspect ratio shape descriptor had similar means for $83 \%$ of the laboratory pairs, while the compactness descriptor had similar means of $47 \%$ of the laboratory pairs.

Bivariate analysis, a pair-wise approach, can be done on descriptor cumulative distributions; one variable is the descriptor value and the other variable is its position in the cumulative distribution. This was done for three descriptors, the Feret diameter, ECD, and the aspect ratio (Table 6). About half of the pairs found to have similar size descriptor means (ANOVA) had similar cumulative distributions (bivariate analysis). Over $50 \%$ of the aspect ratio distributions had similar means. Bivariate analysis, which considers the entire empirical distribution, is more rigorous than ANOVA, which compares only the descriptor means.

As shown by Eqs. (2) and (3), the shape descriptors are ratios of size descriptors. For a descriptor that is a ratio of size descriptors, calibration errors might have a much smaller effect, possibly leading to pairs of laboratories that have similar shape descriptors. Therefore, low numbers of lab pairs with similar size descriptors but high numbers of lab pairs with similar shape descriptors might be due to calibration differences between laboratories.

\subsection{Fitting reference distributions to descriptor data}

The reference models were used empirically in this study; they should be considered when there is little prior experience with mathematical descriptions of a specific sample. The normal distribution is a classic choice. Lognormal distributions often fit data for aerosols or discrete particles synthesized in the liquid phase. The Weibull distribution has been used to model particle comminution from grinding, milling, and crushing [53]. Distributions can be uni-, bi-, or multi-modal. Production processes for commercial powders can be complex, leading to many factors that affect the final size and shape distributions. A task of this study was to develop tools that can explore comparisons of different reference models to distributions, with concomitant analyses of the fits. Thus, users can quickly evaluate potential models via the measurement uncertainties of fitted parameters and evaluate bi- and multi-modal elements. This method is intended to lead to improved particle characterizations for stakeholders in industry, regulatory agencies, academic institutions, and government laboratories.

3.3.1. Single descriptor distributions-We fitted reference models to cumulative distribution data rather than histogram data. Differential probability distributions lose information when the data are binned, often obscuring the details near the tails of the distributions. In general, parameter values from cumulative distribution fits have lower relative standard errors than those for binned differential distributions. Nonlinear regression 
and maximum likelihood methods can provide estimates for reference distribution parameters and their standard errors. These values can be converted to coefficients of variance (Eq. (4)) and then to measurement uncertainty estimates (Eq. (5)). The preferred reference model distribution for a specific data set would generally have parameter estimates with the lowest relative standard errors. Three reference model distributions are often fitted to descriptor cumulative distribution data: normal, lognormal, and Weibull.

Commercial statistical software can provide the coefficient of determination, $R^{2}$, for the preferred fit, the parameter estimate (mean, $\bar{\chi}$, and standard deviation, $\mathrm{s}$, for example), and the standard error of the parameter estimate $\left(u_{\mathcal{\chi}}{ }^{-}\right.$and $\left.u_{S}\right)$. The relative standard error (RSE, the standard error divided by its statistic) is measure of the confidence in the parameter estimate, with smaller values being better.

3.3.2. Fitting distributions to data-A Shiny App ${ }^{\circledR}$, http://shiny.as.uky.edu:3838/curvefitting-app/, was used to fit distributions to data. Figs. 4 and 5 show histograms with data and models, empirical descriptor distributions, and fitted cumulative distributions for two descriptors, Feret diameter and aspect ratio, from laboratory L1. Feret diameter distributions were typical of size descriptors, which were all fitted with the lognormal reference model as the preferred choice. Fig. 4 shows empirical density and cumulative distributions (upper row of panels), and the reference model fits to the data (lower row of panels). While cthe Shiny App $^{\circledR}$ can fit lognormal distributions directly to data, Fig. 4 shows a normal fit to the logarithm of the Feret diameters. As shown in the lower left panel of Fig. 4, both the fitted density distributions and the empirical histogram are symmetric around the mean size, which is $\ln ($ Feret, $\mathrm{nm})=3.84$ and corresponds to Feret $=46.6 \mathrm{~nm}$. Model predictions are compared with the data in the two lower plots (black curve $=$ empirical smoothed density curve of the data, blue curve $=$ lognormal fit using nonlinear least squares regression, red curve $=$ lognormal fit using maximum likelihood estimates). All size descriptor distributions exhibited bi- or multimodal characteristics, but deviations from lognormal reference models appeared to be modest for most size descriptor datasets. Aspect ratio distributions (Fig. 5) were typical of the two shape descriptors, which were all fitted with the Weibull reference model as the preferred choice. In contrast to the size descriptors, the shape descriptor distributions were clearly multimodal, but more detailed modeling was not done for most data in this study.

3.3.3. Relative standard errors of fitted parameters-Table 7 gives the best-fitting parameter values for the aggregated data along with their relative standard errors (RSEs; the standard error of the statistic divided by its mean value). For all scale descriptors, the lognormal reference model estimates for the scale parameter had relative standard errors less than $0.003 \%$ and width parameter estimates with relative standard errors of $0.07 \%$. The shape descriptors, aspect ratio and compactness, are best fitted with Weibull distributions. The two shape descriptors have scale parameter estimates with relative standard errors less than $0.02 \%$. 


\subsection{Visualization tools}

3.4.1. Quantile plots of single descriptor distributions-We present examples of the fitted distributions of the equivalent circular diameter plus their data for three different cases: (A) a pair of laboratories, L3 and L1, with similar descriptor scales (assessed by ANOVA) and similar descriptor distributions (assessed by bivariate analysis), (B) a pair of laboratories, L3 and L4, with similar descriptor scales but dissimilar descriptor distributions, and (C) a pair of laboratories, L3 and L7, for which both the descriptor scales and descriptor distributions are dissimilar. In a quantile plot for a lognormal size distribution model, $X$ equals the natural $\log$ of the size descriptor (ECD in this case) and $Y$ is either the log-normal model of the cumulative distribution or the inverse transpose of the empirical cumulative distribution data. One model line for both the L1 and L3 datasets is shown in gray. These two data-sets have similar scale and width parameters and the models represent the data well for more than two quantiles above and below the mean. The mean value is the value of the $X$ variable when $Y=0$. For clarity, the model lines for the L4 and L7 datasets are not shown in Fig. 6.

For Case A, the L1 and L3 datasets are well-represented by their models over a five quantile range, $-2.5 Y$ to $+2.5 Y$. For Case B (L3 and L4), the lognormal scales of the descriptor differ by $\sim 3 \%$. The different widths of the distributions can easily be visualized as differences in slope on the quantile plots. The model for the L4 data fails for values of $Y<$ -1.5 and $Y>2$. For Case C (L3 and L7), the log-normal scales differ by $\sim 4 \%$ and the distributions clearly have different widths (or slopes). In general, none of the pairwise comparisons of lognormal distributions with statistically different mean values had statistically similar descriptor distributions via bivariate analysis. Thus, ANOVA and bivariate analysis can provide two levels of statistical correspondence as an aid for differentiating between datasets.

3.4.2. Relative residual deviations between model and data-Fig. 7 compares the relative errors, reported as a percentage, between data and model for the $D_{\text {ecd }}$ datasets shown in Fig. 6. The relative residual deviations between the data and models is less than $\pm 5 \%$ over the data range, $2.5<X=\ln \left(D_{\mathrm{ecd}}\right)<4.5$ or $12.2 \mathrm{~nm}<D_{\mathrm{ecd}}<90 \mathrm{~nm}$. For the length descriptors, the lognormal model represents the data well.

As shown in Fig. 5 for the L1 dataset, the aspect ratio descriptor is multi-modal. While unimodal models can be used to fit such data, the parameters of multimodal fits may have lower relative standard errors and lower residual deviations between the model and the data. A bimodal fit to the aspect ratio data in Fig. 5 has been done using two Weibull distributions and a weighting factor that describes the contribution of each to the overall distributions. Five parameters are required, i.e., scale and width parameters for each distribution plus the weighting factor. Fig. 8 shows the relative residual deviations of normal, Weibull, and bimodal Weibull fits to the aspect ratio data of laboratory L1. Compared to the normal distribution, the Weibull model is generally closer to the data over the entire aspect ratio range. The average of the relative residual deviations is $9.7 \%$ for the normal distribution and $5.3 \%$ for the Weibull distribution. While the fitting parameters have similar relative standard errors, the Weibull distribution appears to describe the data better. 
Even better correspondence between model and data can be achieved by fitting a bimodal model to the data. The bimodal Weibull model has an average relative residual deviation of $1.8 \%$, which may justify estimating five parameters rather than 2 . The relative residual deviation plot is another tool that can be used to interpret distribution data: it helps define the descriptor range over which the model fits the data and complements the measurement uncertainties of the fitted parameters.

\subsection{Descriptor - descriptor distributions}

Since titania primary crystallites are asymmetric, pairs of size and shape descriptors can provide important information on their morphology. A specific manufacturing method is likely to generate specific crystallite morphologies. These can be compared for statistical similarity using bivariate analysis.

\subsubsection{Non-parametric comparison of descriptor-descriptor distributions-}

Nanoparticles in commercial products are expected to have variations in size and shape. These, in turn, may affect their performance properties in applications. Two-dimensional plots of descriptors, such as Feret/minFeret (a size-size distribution) or Feret/aspect ratio (a size shape distribution), can be used to explore particle morphology features. Four laboratories reported datasets with similar means as evaluated by pair-wise ANOVA: L1, L2, L3, and L8. There are six unique descriptor pairs of the choice, equivalent circular diameter, Feret diameter, aspect ratio, and compactness. Table 8 shows the bivariate $\mathrm{p}$-values for four sets of size-shape distribution pairs and one size-size distribution pair. Generally, the dataset pairs have distributions similar to each other based on the bivariate test (three pairs, shown in italics, had p-values slightly less than 0.05). All other pairs generated bivariate p-values less than 0.001. Any of these sets of size-shape or size-size descriptors could be used to identify pairs with similar data as assessed by the bivariate test.

3.5.2. Size-size distributions-As show in Table 8, the Feret/minFeret size-size distribution has $p$-values greater than 0.05 for all six laboratory pairs in Table 8 . These results suggest that the Feret and minFeret diameters might be strongly correlated. Fig. 9 shows this size-size distribution for the Lab L3 dataset. The size-size centroids are plotted as $\left(\right.$ Feret $\left._{(\mathrm{ave})}, \operatorname{minFeret}_{(\mathrm{ave})}\right)$. Four datasets were statistically similar based on their pair-wise ANOVA $p$-values $>0.05$ and are shown as diamonds on Fig. 9. Centroids for the remaining datasets are shown as closed circles. A simple correlation is shown the solid black line:

$$
\operatorname{minFeret}=b \cdot \text { Feret }
$$

The $R^{2}$ value for this correlation is 0.886 , where $b=0.602 \pm 0.018$, a relative standard error of $3 \%$.

An alternative model is plotted in Fig. 7 as the dashed line: 


$$
\text { minFeret=aspect } \text { ratio }_{\text {grand }} \text { mean } \cdot \text { Feret }
$$

which assumes that the grand mean of the aspect ratio for the ILC should be a reasonable value for $b$ in Eq. (3). The grand mean of the aspect ratio is 0.632 . The fitted coefficient, $b$, and the grand mean of the aspect ratio are similar, consistent with a hypothesis that differences in the size parameters between datasets for different laboratories could be due to differences in calibration. Comparing ratios of size descriptors, i.e., shape descriptors, should reduce the impact of calibration differences.

3.5.3. Size-shape distributions-Size-shape distributions can be constructed, but do not yield power law descriptor correlations with high $R^{2}$ values for this ILC. Bivariate analysis can be used to compare size-shape distributions reported by different labs. This approach does not rely on assuming reference distributions and has a number of potential applications. However, it does not provide parameters that can be used for measurement uncertainty assessments.

\subsection{Measurement uncertainty assessment}

3.6.1. Coefficients of variation and measurement uncertainty-Measurement uncertainty of ILC means is usually reported for the sizes of certified reference materials. A typical approach [9] is to find the arithmetic mean for each dataset, compute the arithmetic mean and standard deviation of all the dataset means (generating the grand mean and its standard deviation), use Eq. (4) to compute the coefficient of variance, and use Eq. (5) to compute the measurement uncertainty of the particle. The value for $k$ in Eq. (5) is usually taken to be 2 , and for the present study, $n=9$, corresponding to the number of datasets (observations). This procedure starts with an implicit assumption that the descriptor (size) is normally distributed. In addition, the width of the distribution is not assessed across the ILC.

Two methods have been used to compute measurement uncertainties for this ILC. The first method (procedure A) follows the approach for certified reference materials outlined above and adds an assessment of the measurement uncertainty due to the width of the distribution. In Procedure A, the size and shape populations are modeled using the scale and width parameters fitted to a normal distribution. The second method (procedure B) is based on the scale and width parameters for the preferred reference distribution. In both procedures, the grand means and standard deviations of these parameters are then used to compute coefficients of variation for all descriptor distributions (Eq. (1)), which are used to estimate measurement uncertainties (Eq. (2)). In Procedure B, the size distribution is modeled using a lognormal distribution and the shape distribution is modeled using a Weibull distribution. Tables of values for the statistics, coefficients of variation, and measurement uncertainties for all size and shape descriptors are given in the Online Resource.

3.6.2. Size parameter measurement uncertainties-Table 9 presents the results for one size descriptor and one shape descriptor, the primary crystallite equivalent circular diameter and the aspect ratio, respectively. The primary crystallite equivalent diameter is often reported for aggregated titania samples, and we have shown that the lognormal 
distribution provides a better fit to the data than the normal distribution. Analysis of measurement uncertainty values for the pairs, normal vs. lognormal, is one of several ways to compare the various models and datasets. The normal distribution scale factor has a measurement uncertainty 2.3 times that of the lognormal scale factor. The normal distribution width factor has a measurement uncertainty 1.4 times that of the lognormal scale factor. These results confirm that the lognormal choice as the data model is preferred to the normal distribution.

The coefficients of variation for the size descriptors of the full ILC are significantly larger than those reported by Rice for a gold nanoparticle certified reference material [15]. Since a key objective of this certified reference material is to provide a known standard for size, its coefficient of variation for the size descriptor should be relatively small. Reference distributions often fail to represent the data well for very low and very high values of the descriptor (data ranges outside of $\pm 2 \sigma$ from the mean), so the full data should show higher uncertainty values than the model. Of course, the modeled distributions will be easier to use over their ranges of application.

3.6.3. Shape parameter measurement uncertainties-The aspect ratio data (Fig. 3) is multimodal and is not fitted well by the unimodal models reported here. Based on the measurement uncertainties of the aspect ratio parameters, we might favor the normal distribution as being preferred. However, the residual deviation plot (Fig. 8) suggests that the Weibull distribution should be preferred. This finding raises an important point. Using measurement uncertainty of descriptor model parameters alone is not sufficient for full analysis of data quality. Rather, measurement uncertainties should be combined with tools for visualizing the full distribution and its residual deviations. These complementary tools should be used together for selecting appropriate models and assessing data quality.

\section{Summary and conclusions}

This project developed methods for measuring and analyzing primary crystallite size and shape distributions of an aggregate using transmission electron microscopy. Available sample preparation, instrument factors, image acquisition, and particle analysis techniques were modified to meet the measurement challenges of this commercial material. Statistical protocols were used to distinguish between datasets, to select descriptors with similar means, to fit reference models to distribution data, and to compare two-dimensional distributions. There were significant interlaboratory variations with respect to descriptor mean values and fitted parameter estimates of their reference models. The study determined that: 1) calibration method and imaging software did not seem to affect data quality, and 2) some datasets had similar single descriptor means, fitted parameter estimates, and size-size and/or size-shape (two dimensional) distributions. Some evidence indicates that dataset differences in size descriptor means may be due to absolute differences in calibrations. While the equivalent circular diameter, $\mathrm{D}_{\mathrm{ecd}}$, is now a common choice for characterizing titania primary crystallite size, other size descriptors would also have reasonable reproducibility, as shown in the data tables in the Supplementary Material. 
For all datasets, size descriptor distributions appear to conform well to lognormal distributions rather than normal distributions. Scale and width parameters of the aspect ratio shape descriptor have fairly similar measurement uncertainty values for either the normal or the Weibull distributions. However, comparing the residual deviations of the models from the data suggests that the Weibull is preferred. This result suggests that using measurement uncertainty as the only metric by which to judge data quality may not be sound. Rather, it should be used along with visualization tools that can compare model predictions to the data. This general approach can be used by stakeholders for evaluating descriptors of particle size and shape distributions of commercial materials.

\section{Supplementary Material}

Refer to Web version on PubMed Central for supplementary material.

\section{Acknowledgments}

The authors thank Toshiyuki Fujimoto and Naoyuki Taketoshi of AIST for their advice and counsel with respect to ISO/TC229 needs and requirements of this study. The authors also thank the following researchers at NIST for their useful critiques regarding this ILC: Angela Hight Walker, Jeff Fagan, Vince Hackley, and John Bonevich. The work by AIST was part of the research program of "strategic international standardization acceleration projects" supported by the Ministry of Economy, Trade, and Industry (METI) of Japan. The work by BAM on this project was supported by the SETNano-Metro Seventh Framework Programme project (project number 604577; call identifier FP7-NMP-2013_LARGE-7). The work by KRISS was part of the project, "Nano Material technology Development Program (2014M3A7B6020163) of MSIP/NRF. The findings and conclusions in this report are those of the authors and do not necessarily represent the views of the National Institute for Occupational Safety and Health.

\section{References}

1. ISO. ISO 9276-1 Representation of results of particle size analysis - Part 1: Graphical representation. ISO; Geneva: 1998.

2. ISO. ISO 9276-2 Representation of results of particle size analysis - Part 2: Calculation of average particle sizes/diameters and moments from particle size distributions. ISO; Geneva: 2001.

3. ISO. ISO 9276-3 Representation of Results of Particle Size Analysis - Part 3: Fitting of an Experimental Cumulative Curve to a Reference Model. ISO; Geneva: 2008.

4. ISO. ISO 9276-5 Representation of Results of Particle Size Analysis Part 5: Methods of Calculation Relating to Particle Size Analyses using Logarithmic Normal Probability Distribution. ISO; Geneva: 2005. p. 12

5. ISO. ISO 9276-6 Representation of Results of Particle Size Analysis - Part 6: Descriptive and Quantitative Representation of Particle Shape and Morphology. ISO; Geneva: 2008. p. 23

6. ISO. ISO 5725-1:1994(en) Accuracy (trueness and precision) of Measurement Methods and Results — Part 1: General Principles and Definitions. ISO; Geneva, Switzerland: 1994.

7. ISO. ISO 5725-2:1994(en) Accuracy (trueness and precision) of Measurement Methods and Results — Part 2: Basic Method for the Determination of Repeatability and Reproducibility of a Standard Measurement Method. ISO; Geneva, Switzerland: 1994.

8. ISO. ISO 13322-1 Particle Size Analysis - Image Analysis Methods - Part 1: Static Image Analysis Methods. ISO; Geneva: 2004. p. 39

9. NIST, Report of Investigation. Reference Material 8012 Gold nanoparticles, Nominal $30 \mathrm{~nm}$ Diameter. National Institute of Standards and Technology; Gaithersburg, MD: 2007. p. 10

10. Kestens, V., Roebben, G. Certification Report - The Certification of Equivalent Diameters of a Mixture of Silica Nanoparticles in Aqueous Solution: ERM-FD102. IRMM; Geel, Belgium: 2014.

11. Masuda H, Iinoya K. Theoretical study of the scatter of experimental data due to particle-sizedistribution. J Chem Eng Jpn. 1970; 4:60-66. 
12. Masuda H, Gotoh K. Study on the sample size required for the estimation of mean particle diameter. Adv Powder Technol. 1999; 10:159-173.

13. Yoshida H, Mori Y, Masuda H, Yamamoto T. Particle size measurement of standard reference particle candidates and theoretical estimation of uncertainty region. Adv Powder Technol. 2009; 20:145-149.

14. Yoshida H, Yamamoto T, Fukui K, Masuda H. Theoretical calculation of uncertainty region based on the general size distribution in the preparation of standard reference particles for particle size measurement. Adv Powder Technol. 2012; 23:185-190.

15. Rice SB, Chan C, Brown SC, Eschbach P, Han L, Ensor DS, Stefaniak AB, Bonevich J, Vladar AE, Walker ARH, Zheng J, Starnes C, Stromberg A, Ye J, Grulke EA. Particle size distributions by transmission electron microscopy: an interlaboratory comparison case study. Metrologia. 2013; 50:663-678. [PubMed: 26361398]

16. VAMAS. VAMAS Guidelines for the Design and Operation of Interlaboratory Comparisons (ILCs). VAMAS; Washington, DC: 2001. p. 4

17. ISO. ISO 5725 Accuracy (trueness and precision) of Measurement Methods and Results - Part 1: General Principles and Definitions. ISO; Geneva: 1998.

18. Boyd, RD., Cuenat, A., Meli, F., Frase, CG., Klein, T., Krumrey, M., Glever, G., Duta, A., Duta, S., Hogstrom, R., Prieto, E. Measurement Good Practice Guide No 119. National Physical Laboratory; 2011. Good practice guide for the determination of the size distribution of spherical nanoparticle samples.

19. Albers P, Maier M, Reisinger M, Hannebauer B, Weinand R. Physical boundaries within aggregates - differences between amorphous, para-crystalline, and crystalline structures. Cryst Res Technol. 2015; 50:846-865.

20. Pratsinis SE, Zhu W, Vemury S. The role of gas mixing in flame synthesis of titania powders. Powder Technol. 1996; 86:87-93.

21. Maira AJ, Yeung KL, Lee CY, Yue PL, Chan CK. Size effects in gas-phase photo-oxidation of trichloroethylene using nanometer-sized $\mathrm{TiO}_{2}$ catalysts. J Catal. 2000; 192:185-196.

22. Panagiotopoulou P, Christodoulakis A, Kondarides DI, Boghosian S. Particle size effects on the reducibility of titanium dioxide and its relation to the water-gas shift activity of $\mathrm{Pt} / \mathrm{TiO}_{2}$ catalysts. $\mathrm{J}$ Catal. 2006; 240:114-125.

23. Balazs N, Mogyorosi K, Sranko DF, Pallagi A, Alapi T, Oszko A, Dombi A, Sipos P. The effect of particle shape on the activity of nanocrystalline $\mathrm{TiO}_{2}$ photocatalysts in phenol decomposition. Appl Catal B. 2008; 84:356-362.

24. Xin L, Yang C. Study of photocatalytic property of nanosized $\mathrm{TiO}_{2}$, Sanxia Daxue Xuebao, Ziran Kexueban. 2008; 30:68-71.

25. Periasamy VS, Athinarayanan J, Al-Hadi AM, Juhaimi FA, Mahmoud MH, Alshatwi AA. Identification of titanium dioxide nanoparticles in food products: induce intracellular oxidative stress mediated by TNF and CYP1A genes in human lung fibroblast cells, Environ. Toxicol Pharmacol. 2015; 39:176-186.

26. Lin H, Huang CP, Li W, Ni C, Shah SI, Tseng YH. Size dependency of nanocrystalline $\mathrm{TiO}_{2}$ on its optical property and photocatalytic reactivity exemplified by 2-chlorophenol. Appl Catal B. 2006; 68:1-11.

27. Wu N, Wang J, Tafen DN, Wang H, Zheng JG, Lewis JP, Liu X, Leonard SS, Manivannan A. Shape-enhanced photocatalytic activity of single-crystalline anatase $\mathrm{TiO}_{2}(101)$ nanobelts. J Am Chem Soc. 2010; 132:6679-6685. [PubMed: 20420405]

28. Sun B, Smirniotis PG. Interaction of anatase and rutile $\mathrm{TiO}_{2}$ particles in aqueous photooxidation. Catal Today. 2003; 88:49-59.

29. Nakamura R, Nakato Y. Primary intermediates of oxygen photoevolution reaction on $\mathrm{TiO}_{2}$ (rutile) particles, revealed by in situ FTIR absorption and photoluminescence measurements. J Am Chem Soc. 2004; 126:1290-1298. [PubMed: 14746503]

30. Soto K, Garza KM, Murr LE. Cytotoxic effects of aggregated nanomaterials. Acta Biomater. 2007; 3:351-358. [PubMed: 17275430]

31. Horie M, Nishio K, Fujita K, Endoh S, Miyauchi A, Saito Y, Iwahashi H, Yamamoto K, Murayama H, Nakano H, Nanashima N, Niki E, Yoshida Y. Protein adsorption of ultrafine metal oxide and its 
influence on cytotoxicity toward cultured cells. Chem Res Toxicol. 2009; 22:543-553. [PubMed: 19216582]

32. Fadeel B, Garcia-Bennett AE. Better safe than sorry: understanding the toxicological properties of inorganic nanoparticles manufactured for biomedical applications. Adv Drug Deliv Rev. 2010; 62:362-374. [PubMed: 19900497]

33. Nalabotu, SK., Blough, ER. Toxicology of Nanoparticles. CRC Press; 2013. p. 337-354.

34. Kolar M, Mest'ankova H, Jirkovsky J, Heyrovsky M, Subrt J. Some aspects of physico-chemical properties of $\mathrm{TiO}_{2}$ nanocolloids with respect to their age, size, and structure. Langmuir. 2006; 22:598-604. [PubMed: 16401107]

35. Malekfar R, Mihanyar S, Mozaffari M. The back scattering micro-Raman spectroscopy of different crystalline phases of $\mathrm{TiO}_{2}$ nanoparticles produced by sol-gel technique. AIP Conf Proc. 2007; 935:120-125.

36. Zimnyakov DA, Zdrajevsky RA, Yuvchenko SA, Ushakova OV, Angelsky OV, Yermolenko SB. Enhancement of light depolarization by random ensembles of titania-based low-dimensional nanoparticles. J Quant Spectrosc Radiat Transfer. 2015; 152:37-44.

37. Barnard AS. Impact of distributions on the photocatalytic performance of anatase nanoparticle ensembles. J Mater Chem A. 2015; 3:60-64.

38. An H, Pan L, Cui H, Zhou D, Wang B, Zhai J, Li Q, Pan Y. Electrocatalytic performance of Pd nanoparticles supported on $\mathrm{TiO}_{2}-\mathrm{MWCNT}$ for methanol, ethanol, and isopropanol in alkaline media. J Electroanal Chem. 2015; 741:56-63.

39. Zheng X, Kuang Q, Yan K, Qiu Y, Qiu J, Yang S. Mesoporous $\mathrm{TiO}_{2}$ single crystals: facile shape-, size-, and phase-controlled growth and efficient photocatalytic performance. ACS Appl Mater Interfaces. 2013; 5:11249-11257. [PubMed: 24080091]

40. Mandzy N, Grulke E, Druffel T. Breakage of $\mathrm{TiO}_{2}$ agglomerates in electrostatically stabilized aqueous dispersions. Powder Technol. 2005; 160:121-126.

41. Suda Y, Morimoto T, Nagao M. Adsorption of alcohols on titanium dioxide (rutlie) surface. Langmuir. 1987; 3:99-104.

42. Greenwood R, Kendall K. Selection of suitable dispersants for aqueous suspensions of zirconia and titania powders using acoustophoresis. J Eur Ceram Soc. 1999; 19:479-488.

43. Partch R, Brown S. Aerosol and solution modification of particle-polymer interfaces. J Adhes. 1998; 67:259-276.

44. Iguchi, Y., Kuwata, S. Manufacture of Hydrophobic Titanium Oxide Fine Powder. Shinetsu Chemical Industry Co., Ltd; Japan: 1993. p. 5

45. Bonevich, JE., Haller, WK. NIST (Ed) NIST-NCL Joint Assay Protocol, PCC-7 Version 1.1. NISTNCL; Washington, DC: 2010. Measuring the size of nanoparticles using transmission electron microscopy (TEM); p. 13

46. Rizzo ML, Szekely GJ. Energy distance, Wiley Interdiscipl. Rev: Comput Stat. 2016; 8:27-38.

47. Szekely, GJ., Rizzo, ML. Testing for Equal Distributions in High Dimension. Bowling Green State University; 2004.

48. Linsinger, T., Roebben, G., Gilligand, D., Calzolai, L., Rossi, F., Gibson, N., Klein, C.

Requirements of Measurements for the Implementation of the European Commission Definition of the Term "nanomaterial". In: Commission, E., editor. Joint Research Centre, Institute for Reference Materials and Measurements. 2012.

49. McCaffrey JP, Baribeau JM. A transmission electron microscope (TEM) calibration standard sample for all magnification, camera constant, and image-diffraction pattern rotation calibrations. Microsc Res Tech. 1995; 32:449-454. [PubMed: 8563043]

50. Buhr E, Senftleben N, Klein T, Bergmann D, Gnieser D, Frase CG, Bosse H. Characterization of nanoparticles by scanning electron microscopy in transmission mode. Meas Sci Technol. 2009; 20:084025. 084029pp.

51. Klein T, Buhr E, Johnsen KP, Frase CG. Traceable measurement of nanoparticle size using a scanning electron microscope in transmission mode (TSEM). Meas Sci Technol. 2011; 22 094002/094001-094002/094009.

52. Klein T, Buhr E, Johnsen KP, Frase CG. TSEM - a review of scanning electron microscopy in transmission mode and its applications. Adv Imag Electron Phys. 2012; 171:297-356. 
53. Rosin P, Rammler E. The laws governing the fineness of powdered coal. J Inst Fuel. 1933; 7:2936 . 

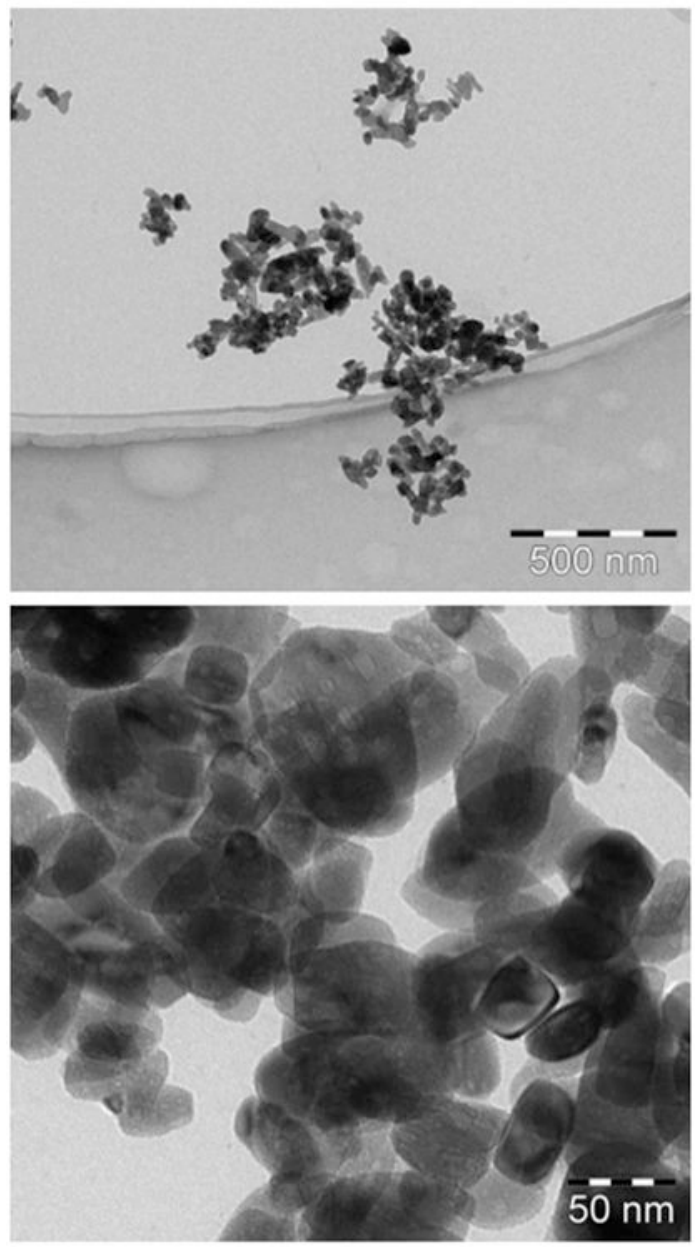

Fig. 1.

TEM images of titania sampled from a water dispersion. (a) Aggregate scale. (b) Primary crystallite scale. 


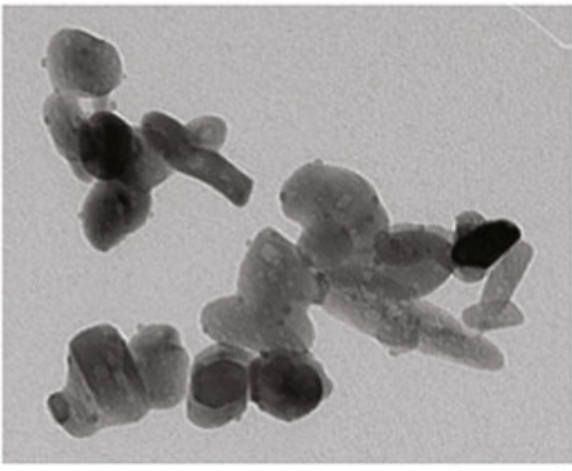

흠.

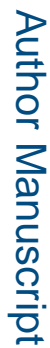

Fig. 2.

Examples of distinguishable primary crystallites.
$50 \mathrm{~nm}$
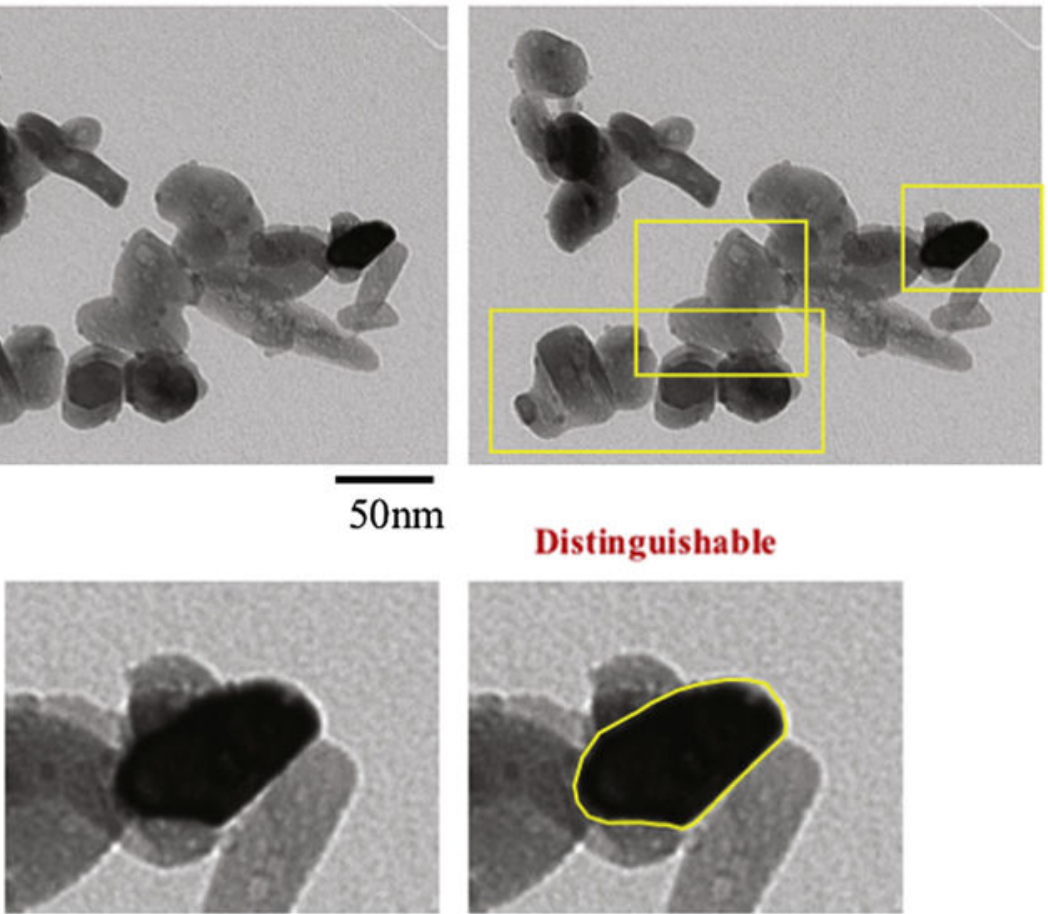

Distinguishable

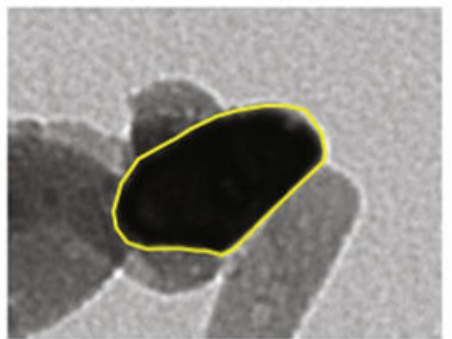

Distinguishable
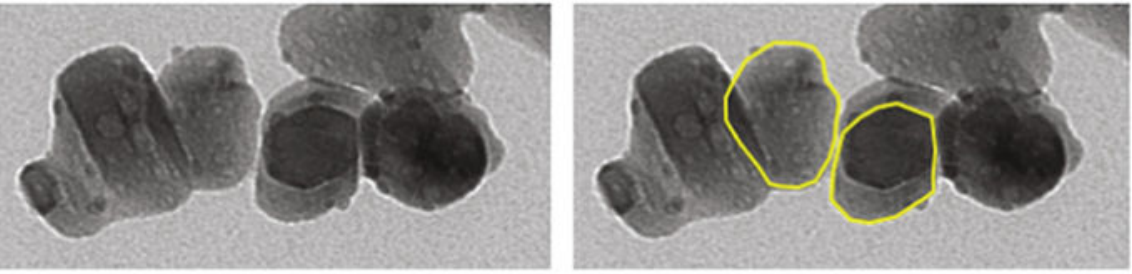

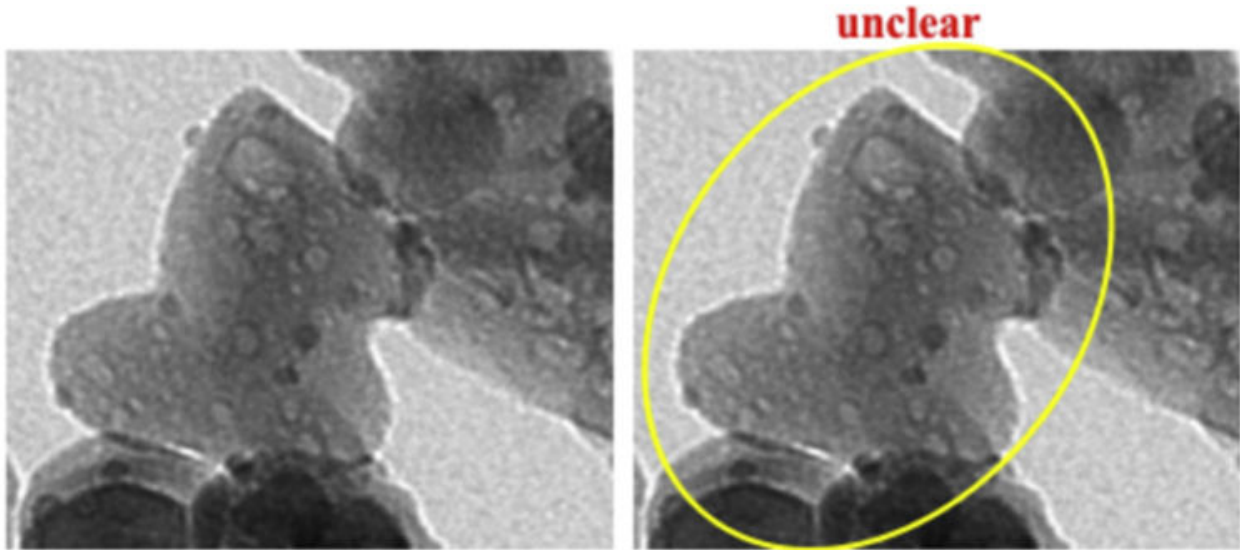

Fig. 3.

Example of primary crystallites that should not be traced. 

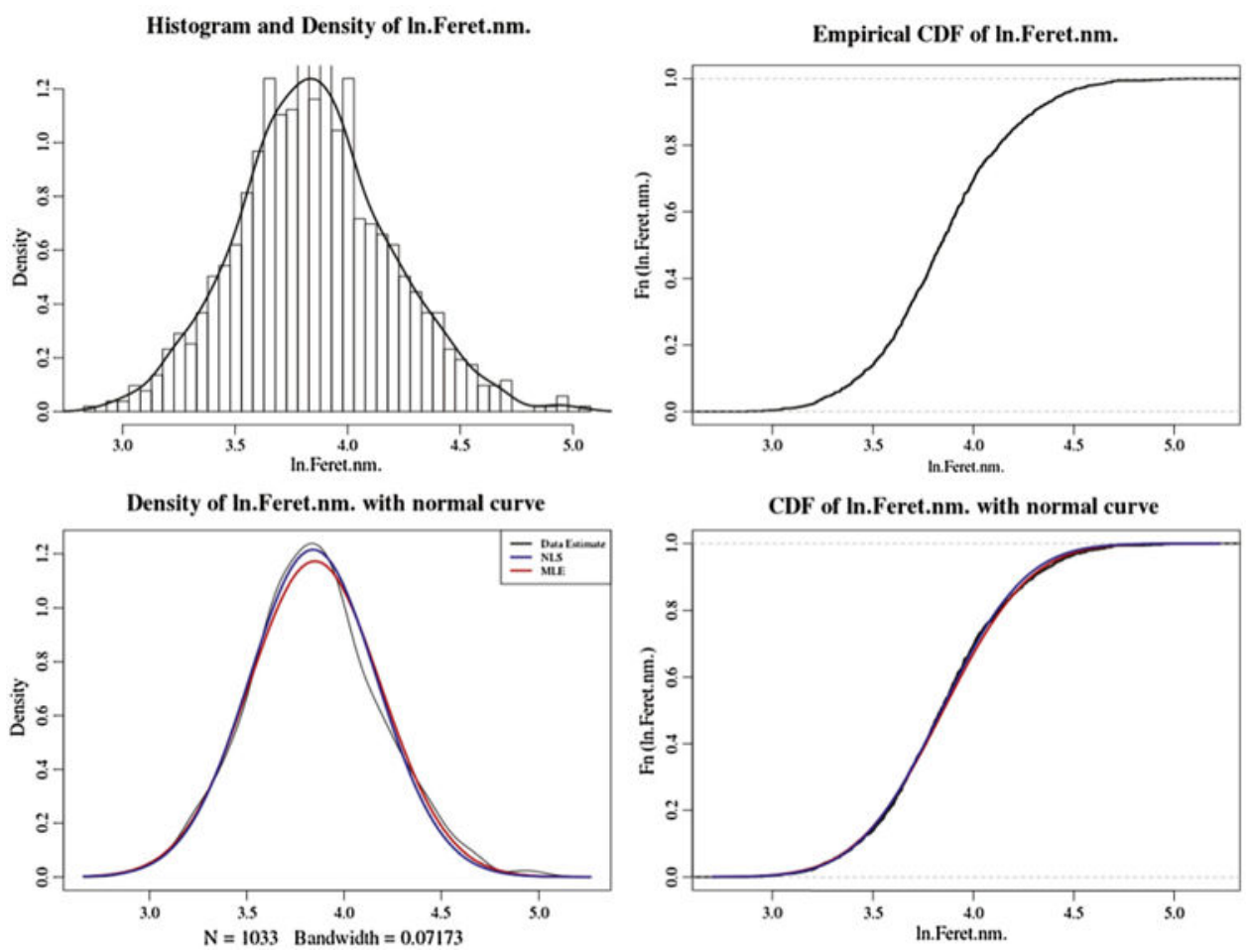

Fig. 4.

Laboratory L1 Feret diameter. Left hand side = density distributions; right hand side = cumulative distributions. Black curve $=$ empirical data; Red curve $=$ maximum likelihood estimate fit; Blue curve $=$ nonlinear regression fit. (For interpretation of the references to colour in this figure legend, the reader is referred to the web version of this article.) 

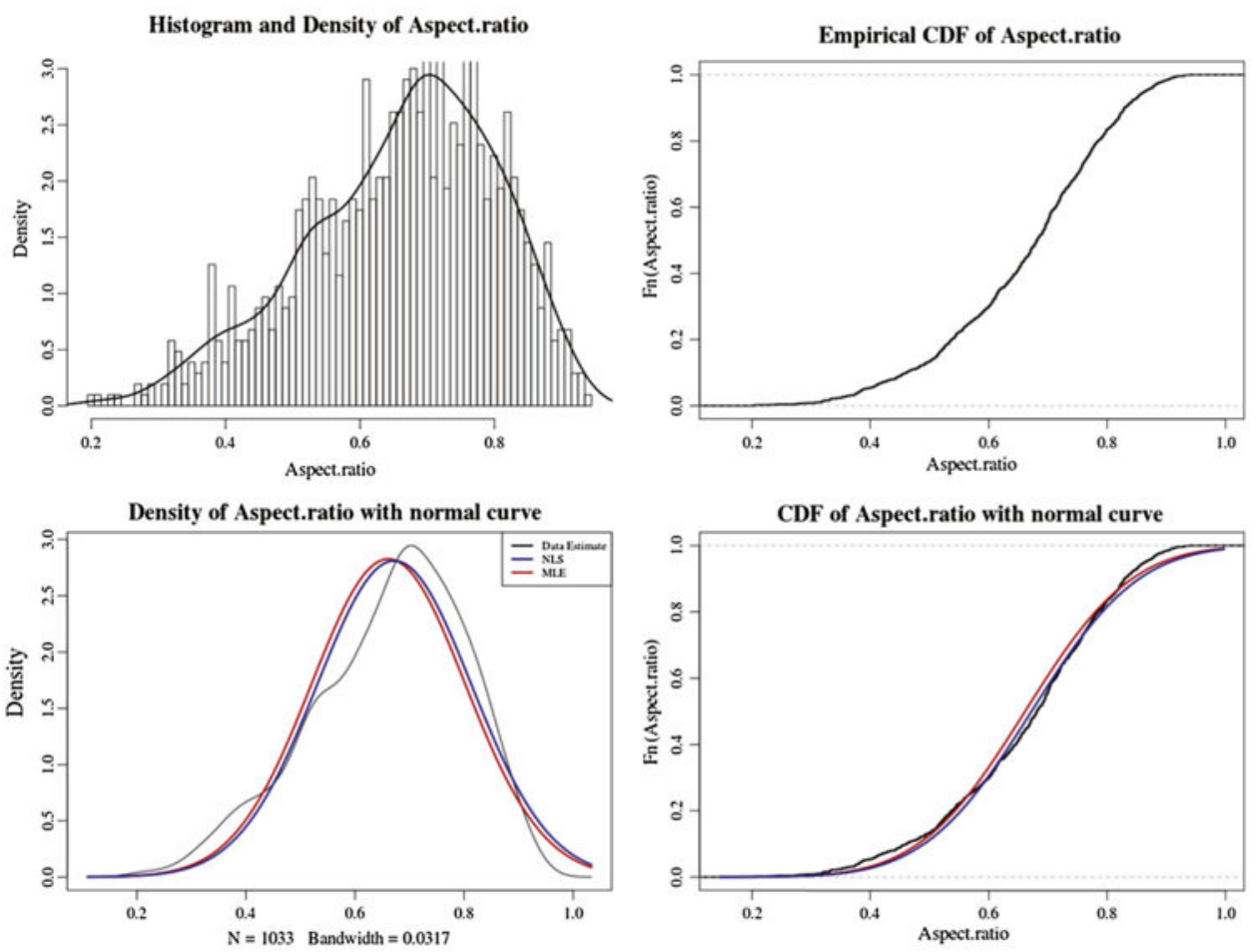

Fig. 5.

Laboratory L1 aspect ratio data. Left hand side = density distributions; right hand side = cumulative distributions; Black curve = empirical data; Red curve $=$ maximum likelihood estimate; Blue curve $=$ nonlinear regression fit. (For interpretation of the references to colour in this figure legend, the reader is referred to the web version of this article.) 


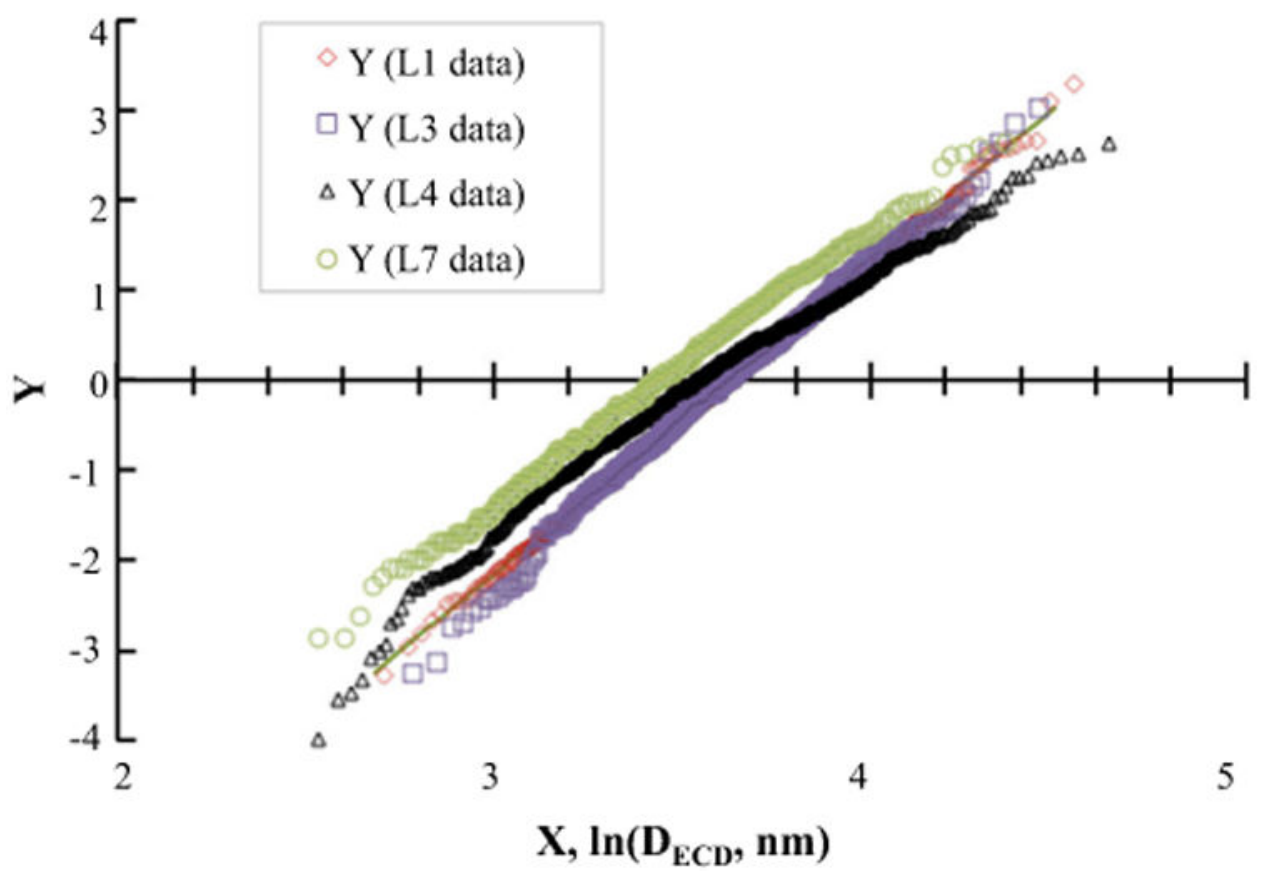

Fig. 6.

Quantile plot comparison of equivalent circular diameters, lognormal reference model. L3 with L1 = similar scale, similar width; L3 with L4 = similar scale, different width; L3 with L7 = different scale, different width. 


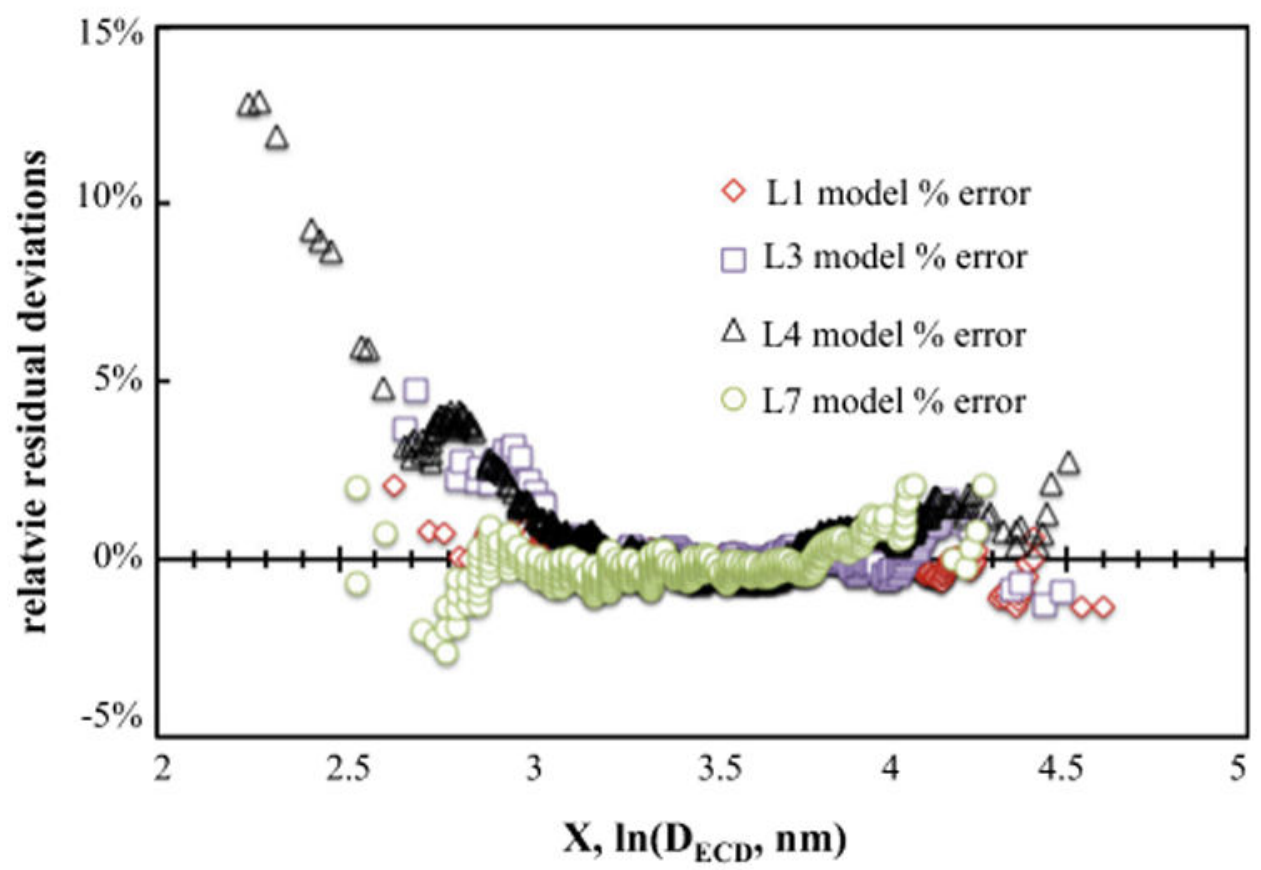

Fig. 7.

Relative residual differences $(\%)$ between equivalent circular diameter data and lognormal models: laboratories L1, L3, L4, and L7. 


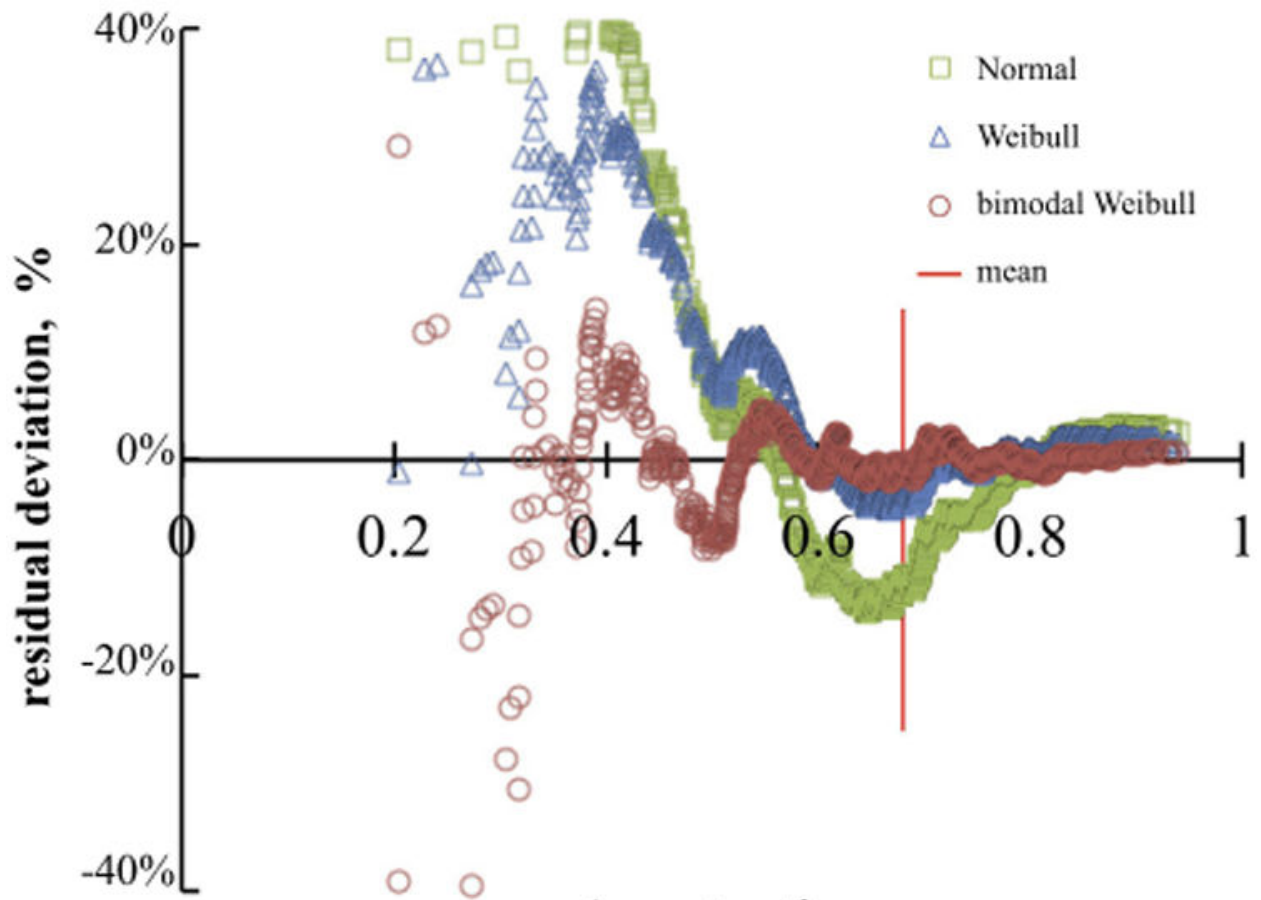

\section{Aspect ratio}

Fig. 8.

Relative residual differences $(\%)$ between aspect ratio data and three models. Data from laboratory 1 . 


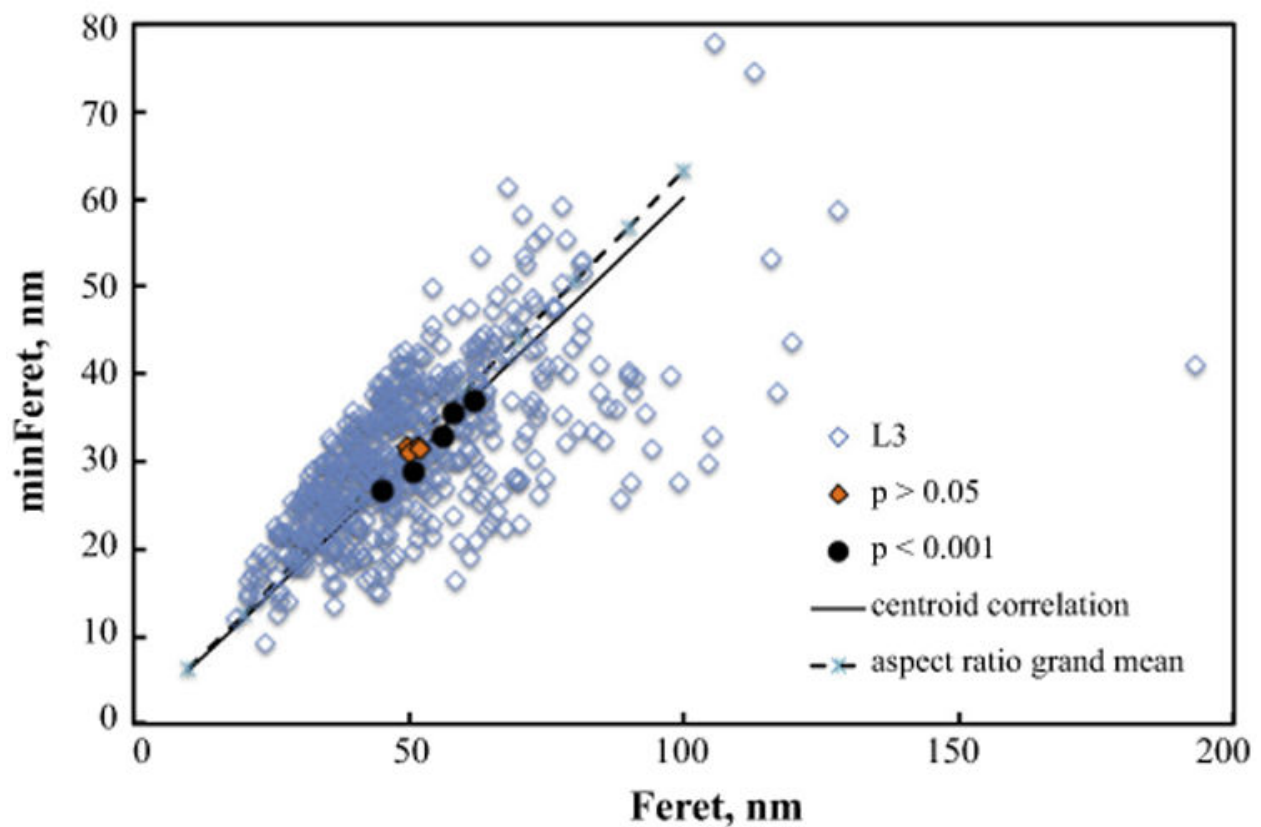

Fig. 9.

Feret/minFeret plot. Open blue diamonds = L3 data; black line diamonds = centroids of statistically similar datasets; black circles = centroids of statistically dissimilar datasets. (For interpretation of the references to colour in this figure legend, the reader is referred to the web version of this article.) 
Table 1

Titania applications dependent on primary crystallite size.

\begin{tabular}{lll}
\hline Application & Preferred primary crystallite size, nm & Reference \\
\hline Lithium ion electrodes & $<1500$ & {$[41]$} \\
Powder cosmetics & $25-200$ & {$[42,43]$} \\
Nanocomposite fibers & 35 & {$[44]$} \\
Dye cell photoanodes & 50 & {$[45]$} \\
Photocatalysts & 20 & {$[46]$} \\
IR-reflective nanocomposites & 41 & {$[47]$} \\
Slurry polishing compound & $10-70$ & {$[48]$} \\
Light emitting diodes & 30 & {$[49]$} \\
Conductive ceramics & $<100$ & {$[50]$} \\
\hline
\end{tabular}




\section{Table 2}

Statistical analysis methods for this ILC.

\begin{tabular}{|c|c|c|c|}
\hline Data element analyzed & Objective & Specific metrics & Statistical method \\
\hline Descriptor datasets & Repeatability or reproducibility & $\begin{array}{l}\text { Grand mean values and standard } \\
\text { deviations Pair-wise p-values }\end{array}$ & $\begin{array}{l}\text { ANOVA of all datasets Pair- } \\
\text { wise ANOVA }\end{array}$ \\
\hline Descriptor distributions & $\begin{array}{l}\text { Repeatability or reproducibility } \\
\text { Coefficient of variance; } \\
\text { measurement uncertainty }\end{array}$ & $\begin{array}{l}\text { Bivariate pair-wise p-values } \backslash \text { Fitted scale } \\
\text { and breadth parameters plus their } \\
\text { standard errors }\end{array}$ & $\begin{array}{l}\text { Bivariate analysis\Curve- } \\
\text { fitting methods: non-linear } \\
\text { regression, maximum } \\
\text { likelihood estimation }\end{array}$ \\
\hline $\begin{array}{l}\text { Size-size and size-shape } \\
\text { distributions }\end{array}$ & Repeatability or reproducibility & Bivariate pair-wise p-values & Bivariate analysis \\
\hline
\end{tabular}




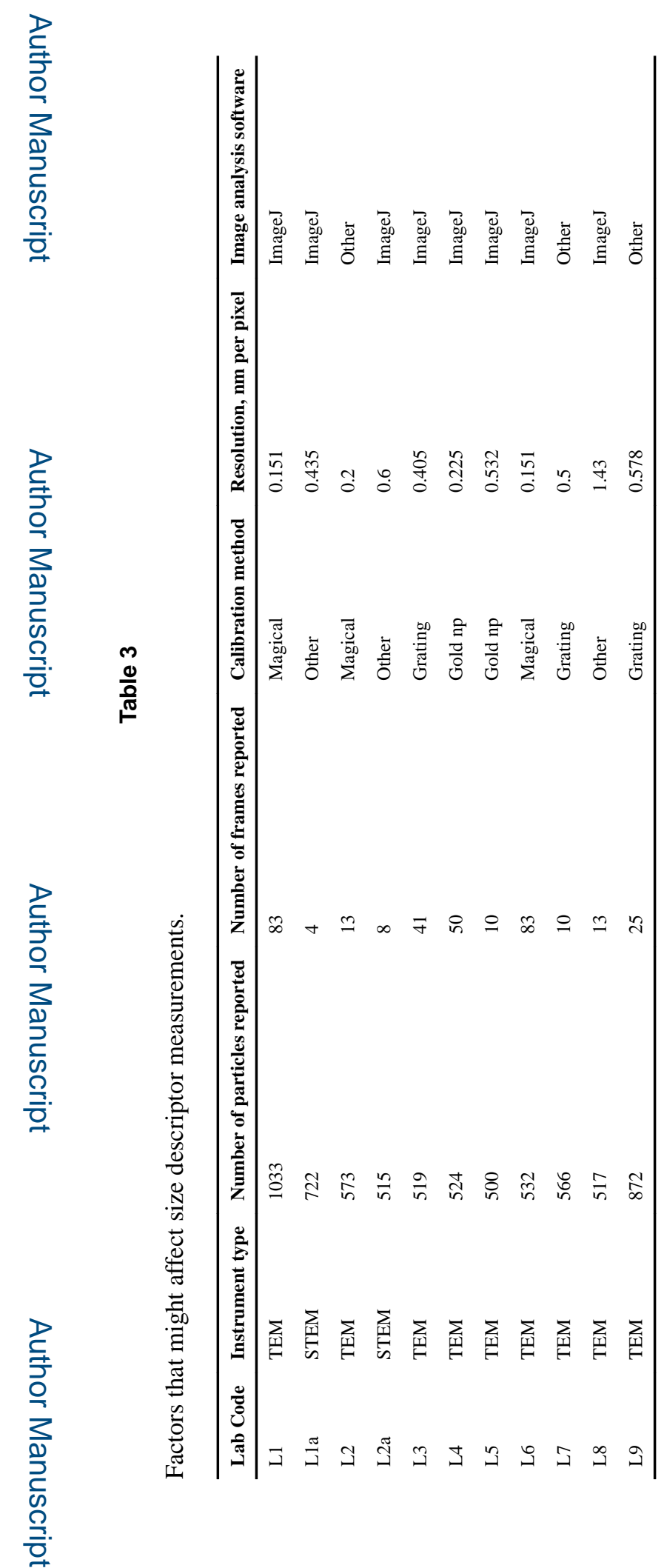

Adv Powder Technol. Author manuscript; available in PMC 2017 December 01. 


\section{Table 4}

Descriptor p-values for calibration method and software categories.

\begin{tabular}{lll}
\hline Descriptor & \multicolumn{2}{l}{$\mathbf{p - v a l u e s}$} \\
\cline { 2 - 3 } & Calibration method & Software \\
\hline Area & 0.0375 & 0.0218 \\
Feret & 0.0394 & 0.0565 \\
minFeret & 0.0538 & 0.225 \\
ECD & 0.353 & 0.144 \\
Aspect ratio & $<0.001$ & 0.061 \\
Compactness & $<0.001$ & $<0.001$ \\
$\%$ similar & $33 \%$ & $67 \%$ \\
\hline
\end{tabular}


Table 5

Estimates of undersized primary crystallites not reported.

\begin{tabular}{llll}
\hline Laboratory & \multicolumn{2}{l}{ Primary crystallite $\mathbf{a r e a , \mathbf { n m } ^ { 2 }}$} & Undersized primary crystallites not reported, \% \\
\cline { 2 - 3 } & Area @ 5\% error & Minimum reported area & \\
\hline L1 & 4.6 & 20 & $\sim 0$ \\
L2 & 8.0 & 80 & $\sim 0$ \\
L3 & 33 & 168 & $\sim 0$ \\
L4 & 10 & 43 & $\sim 0$ \\
L5 & 57 & 142 & $\sim 0$ \\
L6 & 4.6 & 262 & 0.0016 \\
L7 & 50 & 129 & $\sim 0$ \\
L8 & 409 & 414 & 0.30 \\
L9 & 67 & 271 & 0.0024 \\
\hline
\end{tabular}




\section{Table 6}

Pair-wise comparison of descriptors: ANOVA and bivariate methods (36 unique pairs).

\begin{tabular}{llllll}
\hline Descriptor & \multicolumn{2}{l}{ Pair-wise methods } & \multicolumn{2}{l}{} \\
\cline { 2 - 3 } & \multicolumn{2}{l}{ ANOVA, $\mathbf{p}>\mathbf{0 . 0 5}$} & & \multicolumn{2}{l}{ Bivariate, $\mathbf{p}>\mathbf{0 . 0 5}$} \\
\cline { 2 - 3 } \cline { 5 - 6 } & Number & \% & & Number & \% \\
\hline Area & 13 & 36 & - & - \\
Feret & 12 & 33 & 6 & 17 \\
Minferet & 12 & 33 & - & - \\
Decd $_{\text {Aspect ratio }}$ & 30 & 31 & 6 & 17 \\
Compactness & 17 & 47 & - & - \\
\hline
\end{tabular}




\section{Table 7}

Reference model parameters and relative standard errors: size and shape descriptors.

\begin{tabular}{lllll}
\hline & Scale & RSE, scale & Width & RSE, width \\
\hline Size descriptor & \multicolumn{5}{c}{ Lognormal reference model } \\
& 7.053 & $0.00142 \%$ & 0.6310 & $0.01580 \%$ \\
$\ln ($ Area $), \ln \left(\mathrm{nm}^{2}\right)$ & 3.894 & $0.00278 \%$ & 0.3571 & $0.05600 \%$ \\
$\ln ($ Feret $), \ln (\mathrm{nm})$ & 3.426 & $0.00292 \%$ & 0.3231 & $0.06190 \%$ \\
$\ln (\operatorname{minFeret}), \ln (\mathrm{nm})$ & 3.647 & $0.00274 \%$ & 0.3156 & $0.03170 \%$ \\
$\ln \left(\mathrm{D}_{\text {ecd }}\right), \ln (\mathrm{nm})$ & & & & \\
Shape descriptor & & & & \\
& Weibull reference model & \\
Aspect ratio & 0.7104 & $0.0141 \%$ & 4.781 & $0.00274 \%$ \\
Compactness & 0.8216 & $0.0122 \%$ & 9.963 & $0.00853 \%$ \\
\hline
\end{tabular}



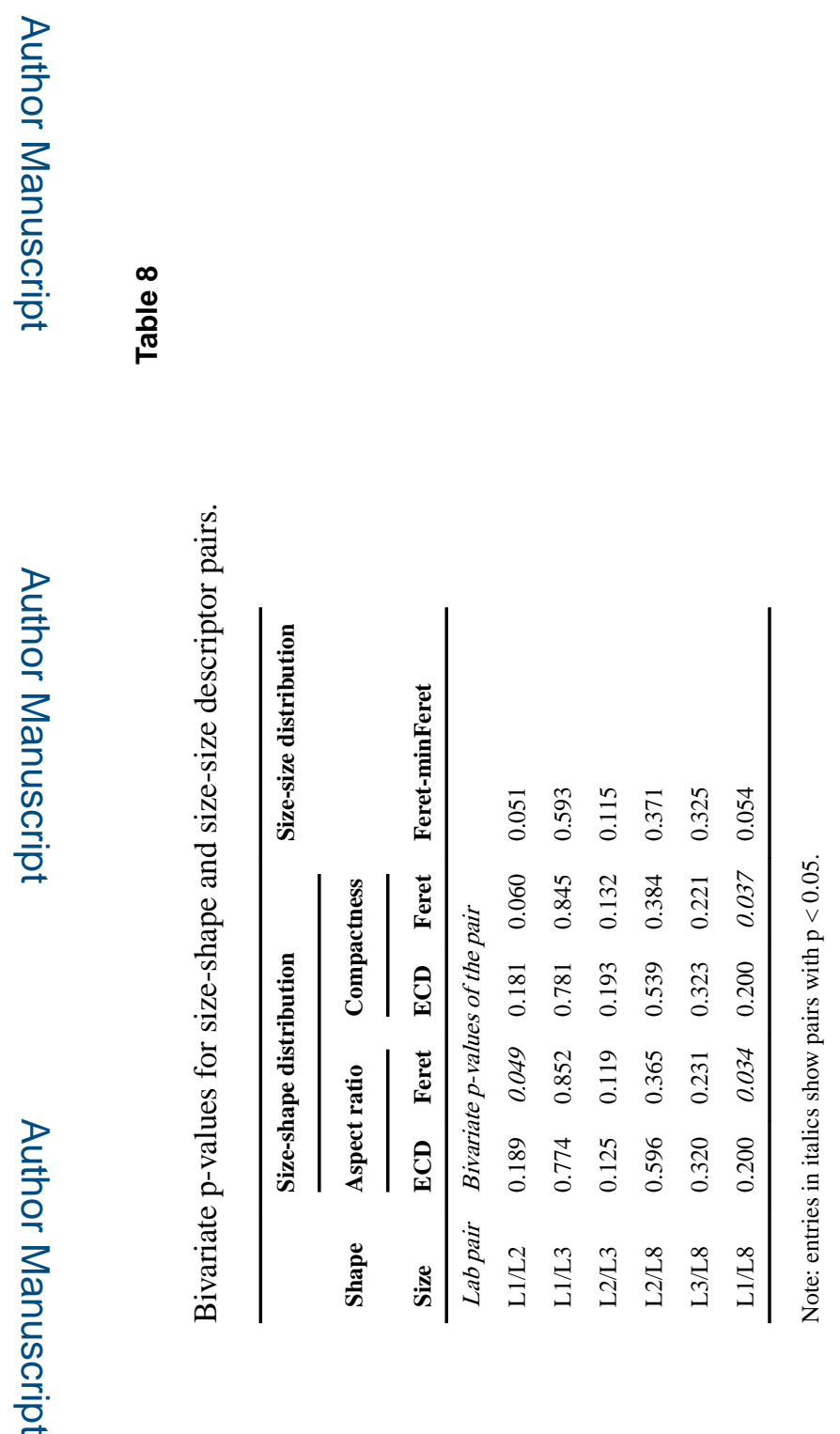

Adv Powder Technol. Author manuscript; available in PMC 2017 December 01. 


\section{로을}

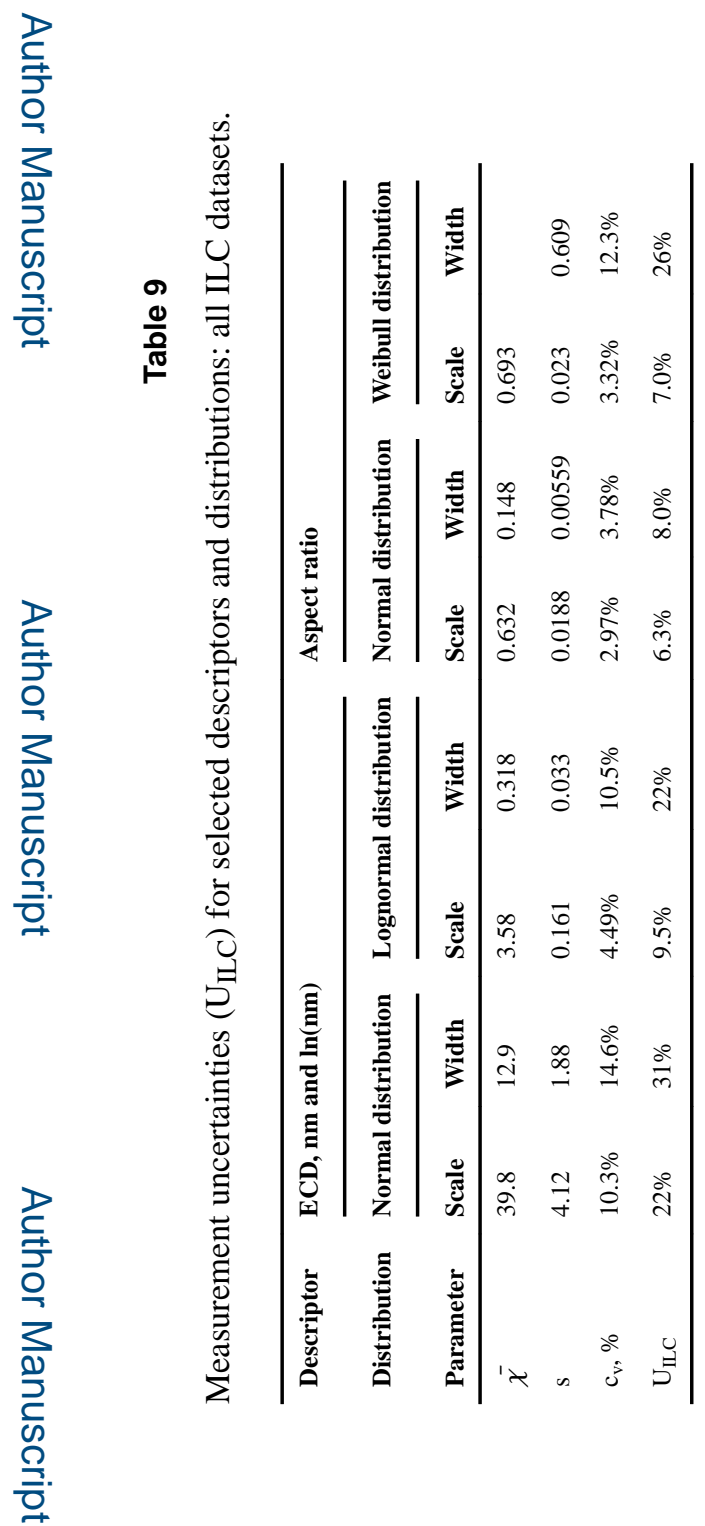

Adv Powder Technol. Author manuscript; available in PMC 2017 December 01. 